\title{
éditionsOCDE
}

\section{LES INÉGALITÉS DE GENRE DANS LES INSTITUTIONS SOCIALES OUEST-AFRICAINES}

\section{NOTES OUEST-AFRICAINES}

Mars 2018 No. 13 



\section{LES INÉGALITÉS DE GENRE DANS LES INSTITUTIONS SOCIALES OUEST-AFRICAINES}

Cette note a été rédigée par

NEJMA BOUCHAMA

GAËLLE FERRANT

LÉA FUIRET

ALEJANDRA MENESES

ANNELISE THIM

Équipe en charge du Programme égalité femmes-hommes du Centre de développement de l'OCDE 


\section{NOTES OUEST-AFRICAINES}

La série Notes ouest-africaines analyse les dynamiques socio-économiques, politiques et sécuritaires que traverse l'Afrique dans une perspective régionale et multidisciplinaire. Elle cherche à stimuler la discussion, rassembler les informations et mieux anticiper les transformations en cours pour les politiques à venir. La série vise à partager des études avec une large audience d'experts, de praticiens du développement, de décisionnaires et de lecteurs avertis. Les Notes sont disponibles en anglais et/ou en français ; les résumés dans les deux langues. Initiées par le Club du Sahel et de l'Afrique de l'Ouest (CSAO) pour éclairer les enjeux ouest-africains, ces analyses sont préparées par son Secrétariat, ses membres et partenaires, les autres départements de l'OCDE, des organisations internationales et autres experts et chercheurs.

En savoir plus sur le Club du Sahel et de l'Afrique de l'Ouest : http://www.oecd.org/fr/csao.

Merci de citer cet ouvrage comme suit:

Bouchama, N., G. Ferrant, L. Fuiret, A. Meneses et A. Thim (2018), « Les inégalités de genre dans les institutions sociales ouest-africaines m, Notes ouest-africaines, $N^{\circ} 13$, Éditions OCDE, Paris.

https://doi.org/10.1787/7357808d-fr

Contact auteur : gaelle.ferrant@oecd.org

ISSN 2415-1149

Les documents de travail de l'OCDE ne doivent pas être présentés comme exprimant les vues officielles de l'OCDE ou de ses pays membres. Les opinions exprimées et les arguments employés sont ceux des auteurs.

Ce document et toute carte qu'il peut comprendre ne préjugent en rien du statut de tout territoire, de la souveraineté s'exerçant sur ce dernier, du tracé des frontières et limites internationales, et du nom de tout territoire, ville ou région.

Les documents de travail exposent des résultats préliminaires ou des travaux de recherche en cours menés par l'auteur/les auteurs et sont publiés pour stimuler le débat sur un large éventail de questions sur lesquelles l'OCDE travaille. Les commentaires sur les documents de travail sont bienvenus et peuvent être adressés au Club du Sahel et de l'Afrique de l'Ouest, OCDE, 2 rue André-Pascal, 75775 Paris Cedex 16, France.

Autorisé pour publication par Laurent Bossard, Directeur, Secrétariat du Club du Sahel et de l'Afrique de l'Ouest (CSAO/OCDE).

(C) OCDE 2018

Vous êtes autorisés à copier, télécharger ou imprimer du contenu OCDE pour votre utilisation personnelle. Vous pouvez inclure des extraits des publications, des bases de données et produits multimédia de l'OCDE dans vos documents, présentations, blogs, sites Internet et matériel d'enseignement, sous réserve de faire mention de la source OCDE et du copyright. Les demandes pour usage commercial ou de traduction devront être adressées à rights@oecd.org. 


\section{RÉSUMÉ}

Les institutions sociales discriminatoires que sont les lois formelles et informelles, normes et pratiques sociales, restreignent les droits et les opportunités d'autonomisation des femmes dans les 17 pays d'Afrique de l'Ouest. De nouvelles lois et mesures sur les droits des femmes et la parité se sont accompagnées de progrès sensibles. Cependant, les institutions sociales discriminatoires continuent à contraindre l'accès des femmes aux ressources productives, à favoriser les atteintes à leur intégrité physique et à restreindre leur pouvoir décisionnel dans les sphères privée et publique. Cela entrave l'accès à l'éducation et aux opportunités économiques des femmes et des filles, réduisant ainsi le potentiel de développement des pays. Les analyses basées sur l'indicateur Institutions sociales et égalité femme-homme (SIGI) du Centre de développement de l'OCDE fournissent aux décideurs politiques et acteurs du développement des éléments probants nécessaires à la conception de politiques et de programmes plus efficaces. Placer les institutions sociales au cœur des réponses politiques ouvre des perspectives nouvelles et durables de promotion de l'égalité femmes-hommes dans les stratégies de développement nationales et régionales.

Mots-clés : femmes, institutions sociales, inégalité des genres, Afrique de l'Ouest

Classification JEL : J16, K38, N37

\section{REMERCIEMENTS}

Cette note a été rédigée à la demande et avec le financement du Club du Sahel et de l'Afrique de l'Ouest. Le Centre de développement de l'OCDE tient à remercier le Secrétariat du CSAO/OCDE pour sa coopération, ses commentaires et son expertise, en particulier Nadia Hamel, qui a coordonné cette note, et Marie Trémolières. Des remerciements particuliers sont adressés à Joël Boutroue, Bathylle Missika et Lorenzo Pavone (Centre de développement de l'OCDE) pour leurs commentaires judicieux et Julie Bousquet pour son soutien. 


\section{TABLE DES MATIÈRES}

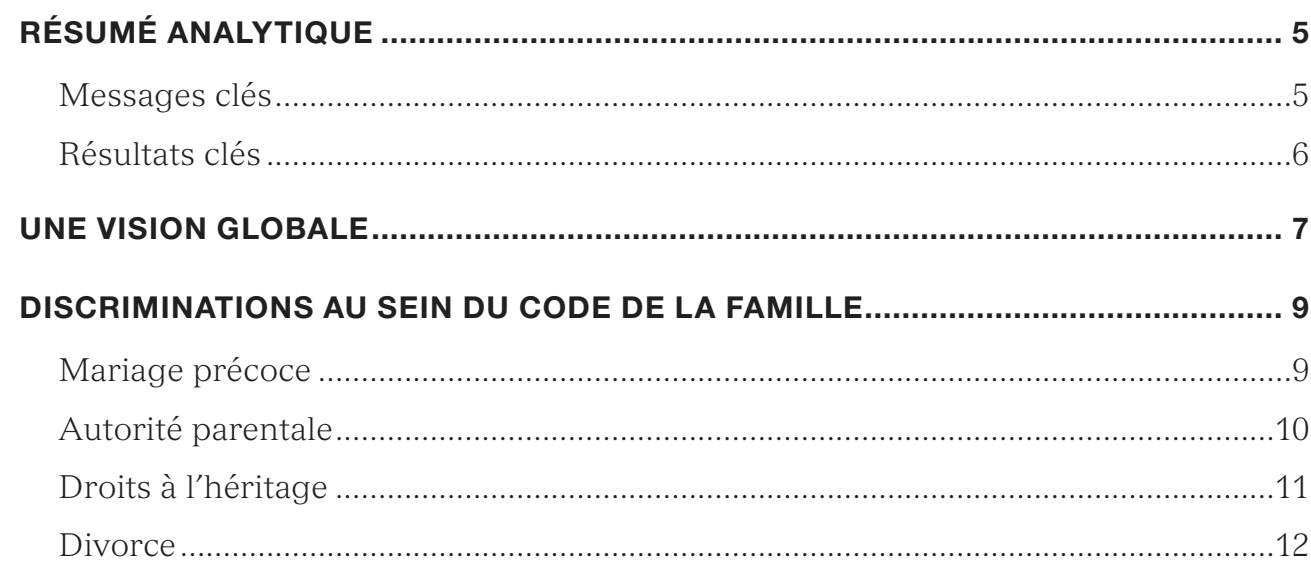

ATTEINTES À L'INTÉGRITÉ PHYSIQUE ...............................................................13

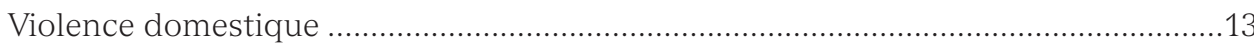

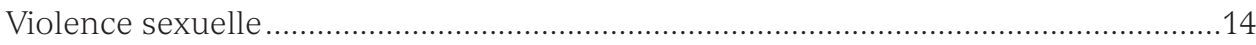

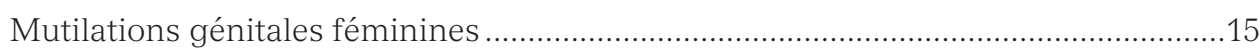

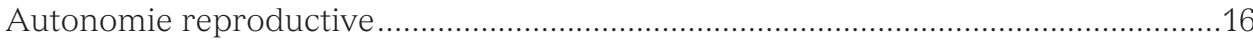

ACCÈS RESTREINT AUX RESSOURCES ET AUX BIENS ............................................17

Accès sécurisé à la terre...........................................................................................17

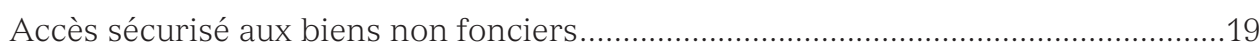

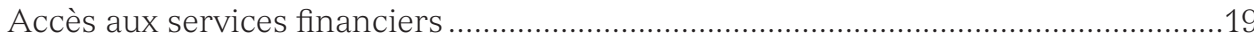

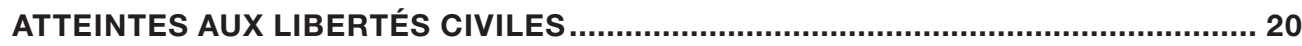

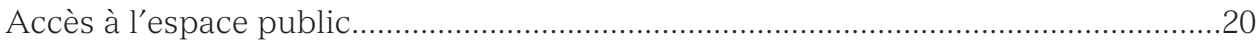

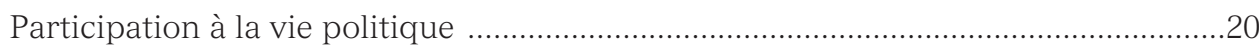

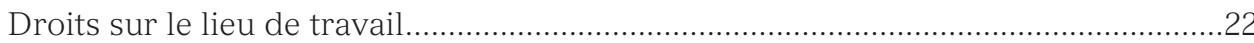

NOTES

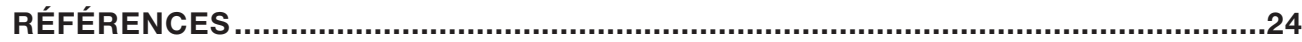

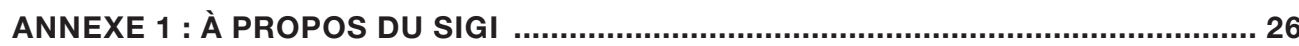

ANNEXE 2 : RÉSULTATS PAR SOUS-INDICATEUR DU SIGI POUR LES PAYS

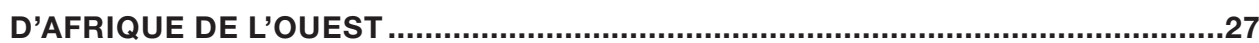

ANNEXE 3 : RATIFICATIONS DES PROTOCOLES MONDIAUX ET RÉGIONAUX SUR

L'ÉGALITÉ FEMMES-HOMMES ...................................................................... 28

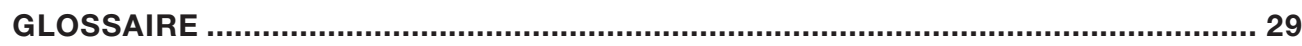




\section{RÉSUMÉ ANALYTIQUE}

C ette note analyse les résultats de l'indicateur Institutions sociales et égalité femmehomme (SIGI) pour 17 pays d'Afrique de l'Ouest ${ }^{1}$ à travers les dimensions « discriminations au sein du code de la famille ), ((atteintes à l'intégrité physique ), ( accès restreint aux ressources et aux biens » et ( atteintes aux libertés civiles ») (Graphique 1)². Elle examine la façon dont les réformes législatives et les efforts de transformation des institutions sociales discriminatoires peuvent se traduire par des changements positifs pour les femmes et leur communauté ; elle identifie les défis qui subsistent. Elle souligne la nécessité d'intégrer la lutte contre les institutions sociales discriminatoires dans les politiques visant à garantir aux femmes et aux filles les mêmes droits et opportunités qu'aux hommes et aux garçons.

Restreindre le champ des possibles et les opportunités offertes aux femmes et aux filles réduit le potentiel de développement économique et humain des pays. Ainsi, au-delà de sa qualité de droit fondamental, la parité représente une opportunité économique majeure : les économies sont plus résilientes, productives et inclusives lorsqu'elles réduisent les inégalités femmes-hommes et soutiennent activement la participation des femmes et des filles dans tous les domaines (Ferrant et Kolev, 2016 ; Banque mondiale, 2012).

En adoptant les Objectifs de développement durable (ODD), en particulier l'objectif $5^{3}$, les gouvernements, et notamment ceux d'Afrique de l'Ouest, se sont engagés à promouvoir la parité, protéger les droits des femmes et éliminer toutes les restrictions auxquelles elles font face tout au long de leur vie. Alors que d'importants progrès ont été accomplis ces dernières années en Afrique de l'Ouest, de nombreux défis restent à relever ; en particulier l'élimination des institutions sociales discriminatoires qui réduisent les opportunités et le bien-être des femmes, et de ce fait limitent le potentiel de développement des économies ouest-africaines.

\section{Messages clés}

1. Intégrer la lutte contre les institutions sociales discriminatoires au centre des politiques sensibles au genre et des stratégies de développement inclusif. La transformation des normes sociales offre d'importantes opportunités de développement aux pays d'Afrique de l'Ouest. S'attaquer aux défis posés par les institutions sociales discriminatoires exige des réponses politiques fondées sur des faits empiriques et des analyses factuelles, adaptées aux différents contextes, et prenant en compte les incidences sexo-spécifiques.

2. Collecter des données empiriques sur l'étendue et la nature des institutions sociales discriminatoires. Mesurer permet de rendre visible l'invisible et de mieux comprendre les obstacles et bonnes pratiques susceptibles de transformer les institutions sociales discriminatoires sur la base des réalités locales. Cela permet aux gouvernements de mieux comprendre les liens entre les institutions sociales discriminatoires, une plus grande parité et le développement. Développer les capacités statistiques et analytiques nationales permettra aux gouvernements de mesurer les discriminations et d'évaluer l'efficacité des politiques. 


\section{Résultats clés}

1. La perte de revenu associée aux discriminations de genre dans les institutions sociales est estimée à 120 milliards de dollars (USD) pour la région. L'égalité femmes-hommes est donc, aussi, un objectif économique. En restreignant l'accès des femmes à l'éducation et à l'emploi, les institutions sociales discriminatoires ont un impact négatif sur la croissance économique et le bien-être. Au niveau mondial, la perte de revenu associée au niveau actuel de discrimination de genre dans les institutions sociales est estimée à 12000 milliards de dollars (USD), l'équivalent de $16 \%$ du PIB mondial actuel (Ferrant et Kolev, 2016).

2. Les normes et les pratiques discriminatoires au sein de la famille accentuent les disparités femmes-hommes. Le mariage précoce limite la capacité des filles à poursuivre leurs études et à obtenir des emplois rémunérés. Être mariée avant 18 ans réduit la probabilité d'achever ses études et augmente les risques en matière de santé. Malgré des signes de réduction de la pratique, $30 \%$ des filles ouest-africaines âgées de 15 à 19 ans sont mariées, divorcées ou veuves (DHS, s.d.) ; la moyenne mondiale étant de $13 \%$.

3. Les atteintes à l'intégrité physique des femmes sont profondément ancrées dans les normes sociales. Malgré les programmes de lutte contre les mutilations génitales féminines (MGF), cette pratique est encore enracinée dans les communautés, y compris chez les femmes, ce qui freine les progrès. La moitié des femmes ouest-africaines ont été victimes de MGF (DHS, s.d.). Les taux les plus élevés (neuf femmes sur dix) touchent la Guinée et le Mali ; dans ces pays, trois femmes sur quatre pensent que cette pratique devrait perdurer (DHS, s.d.).

4. Les différences entre le droit coutumier et le cadre juridique affaiblissent les droits des femmes relatifs à l'accès à la terre. Même là où la loi garantit les droits fonciers des femmes, des coutumes et pratiques discriminatoires limitent leur capacité à posséder la terre, à la contrôler, à en hériter, à la gérer et à prendre des décisions la concernant. Dans sept pays d'Afrique de l'Ouest ${ }^{4}$, les femmes représentent en moyenne $43 \%$ des travailleurs agricoles, mais seulement $8 \%$ des propriétaires terriens (FAO, 2011).

5. La participation politique des femmes progresse en Afrique de l'Ouest, mais reste inférieure à la moyenne mondiale. Sept pays ${ }^{5}$ ont mis en place des quotas aux niveaux national et/ou local pour promouvoir la participation politique des femmes. Néanmoins, dans la région, la proportion de femmes siégeant au parlement reste faible et progresse peu (13\% en 2007, $16 \%$ en 2017). Les disparités entre les pays sont par ailleurs fortes (de $43 \%$ au Sénégal à 6 \% au Nigéria) (UIP, 2017).

Graphique 1

Composition du SIGI

Indicateur " Institutions sociales et égalité femme-homme » (SIGI)
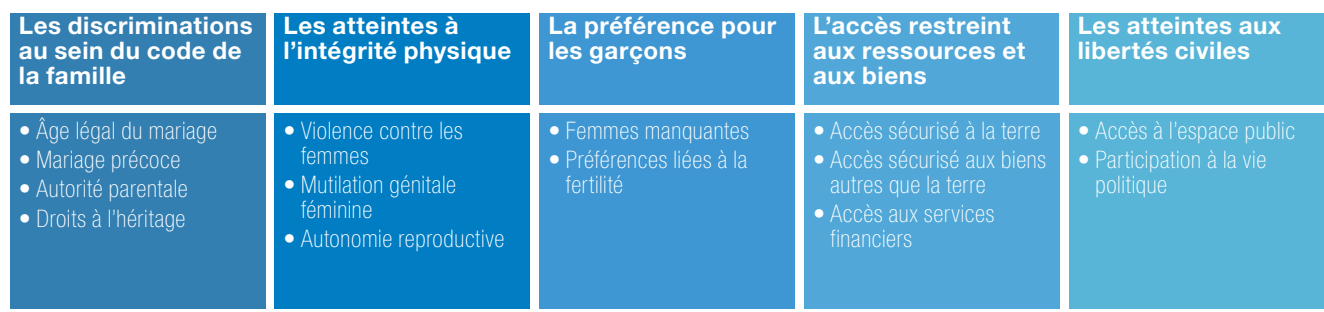


\section{UNE VISION GLOBALE}

- es résultats globaux du SIGI traduisent la faible performance des pays

d'Afrique de l'Ouest dans la réduction des discriminations de genre au sein des institutions sociales :

- Dans trois pays (Guinée-Bissau, Sénégal et Togo), les institutions sociales affichent un niveau de discrimination intermédiaire par rapport à la moyenne mondiale. Ces pays se caractérisent par des cadres juridiques contradictoires marqués par une pluralité de systèmes juridiques (coexistence de la loi écrite et du droit coutumier) et par des pratiques coutumières perpétuant les discriminations.

- $\quad$ Cinq pays (Bénin, Burkina Faso, Côte d’Ivoire, Ghana et Guinée) affichent des niveaux de discrimination élevés. Dans ces pays, les discriminations dans le droit coutumier et les normes et pratiques sociales se traduisent par une protection juridique des femmes inadéquate.

- Le niveau de discrimination est très élevé dans les huit pays restants (Gambie, Libéria, Mali, Mauritanie, Niger, Nigéria, Sierra Leone et Tchad). À des cadres juridiques très discriminatoires et des mécanismes de mise en œuvre médiocres s'ajoutent des pratiques et normes sociales qui négligent les droits des femmes (carte 1) .

Carte 1

Résultats SIGI dans les pays d'Afrique de l'Ouest

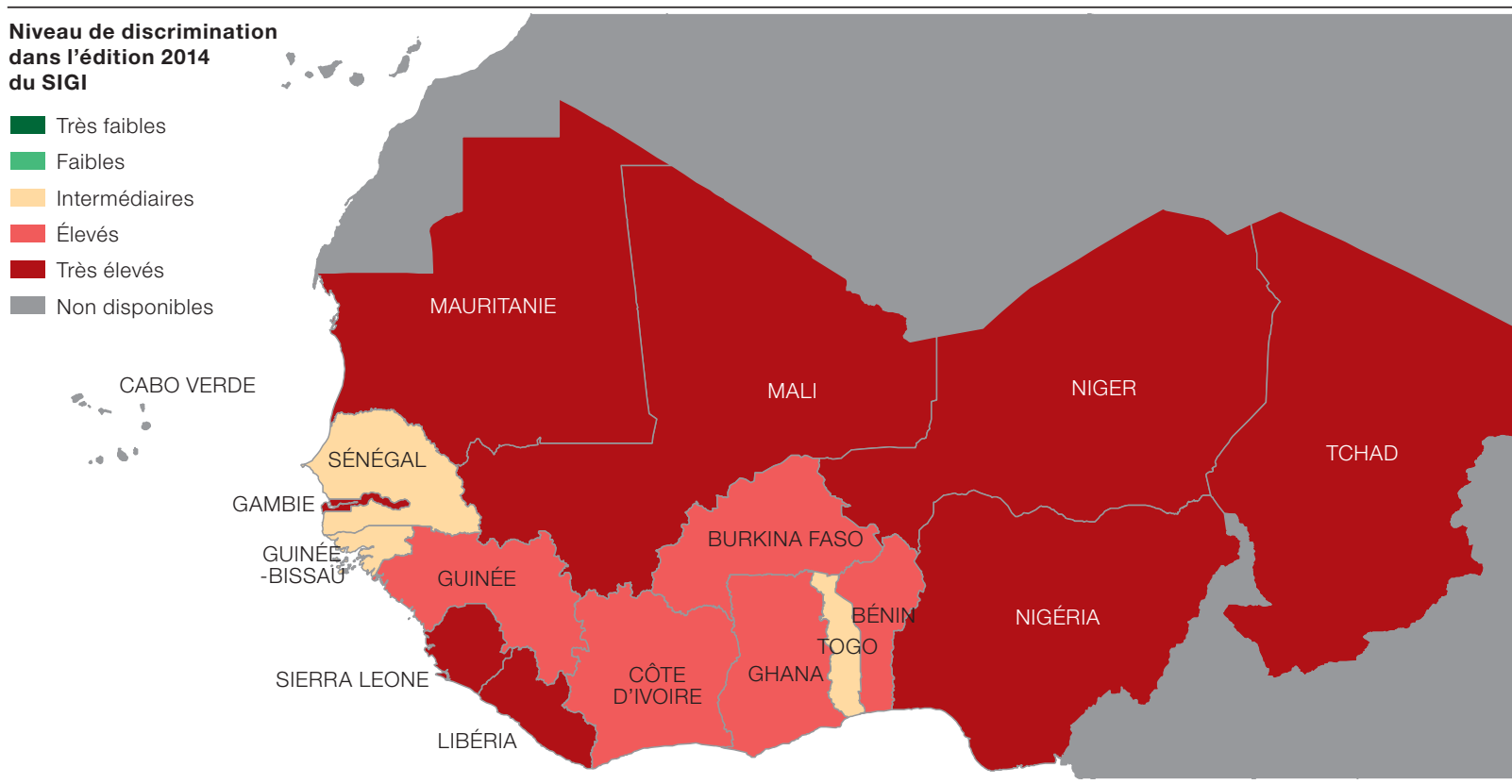

Source : OCDE (2014a), Base de données Égalité homme-femme, Institutions et Développement, http://stats.oecd.org

Les femmes ouest-africaines subissent des niveaux de discrimination très élevés dans trois dimensions (Graphique 2) :

- Discriminations au sein du code de la famille : la moitié des pays de la région conservent des attitudes et pratiques discriminatoires en matière d’héritage, de mariage et de divorce. En outre, les constitutions nationales ne leur reconnaissent pas toujours les mêmes droits qu'aux hommes. Cinq pays (Gambie, Mauritanie, Niger, Nigéria et Tchad) ne garantissent pas les mêmes droits à l'héritage aux veuves et aux veufs. Dans les autres pays, la garantie formelle des droits en la matière est remise en cause par des pratiques traditionnelles ou religieuses exerçant une discrimination à l'encontre des veuves et des filles. Par exemple, au Libéria, les veuves ont les mêmes droits à l’héritage que les veufs, mais des accusations de sorcellerie peuvent être portées contre elles pour les empêcher d'hériter de biens. De telles accusations surviennent souvent lorsque l'époux décédé était plus nanti que sa veuve (Commission de 
l'immigration et du statut de réfugié du Canada, 2013). Dans certains villages de l'ouest de la Côte d'Ivoire, au décès d'un homme qui a seulement des filles, c'est son frère qui hérite de ses biens (FAO, 2011).

- Atteintes à l'intégrité physique : une autre source de préoccupation tient à la faiblesse de la couverture et de la qualité de la protection juridique et des autres mesures contre les violences faites aux femmes (viol, harcèlement sexuel et violence domestique) ainsi que les mutilations génitales féminines (MGF). Il en va de même pour ce qui concerne la santé reproductive. En moyenne, 18 \% des femmes ont été victimes de violence domestique au cours de leur vie au Nigéria, jusqu'à 57 \% au Tchad (OCDE, 2014a). Cette pratique est socialement acceptée : 50 \% des femmes pensent que la violence domestique est justifiée sous certaines conditions.

- Accès restreint aux ressources et aux biens : les droits et l'accès des femmes à la terre, aux biens et aux services financiers sont compromis par des lois et des pratiques discriminatoires qui les empêchent de posséder ces ressources essentielles à leur autonomisation économique et financière. Seuls le Libéria et le Mali ont un cadre juridique garantissant les mêmes droits aux femmes et aux hommes concernant l'accès aux services financiers formels.

Cependant, leurs engagements s'étant traduits en bonnes pratiques, la Mauritanie et le Sénégal ont les meilleures performances de la région en termes de libertés civiles. La Constitution de la Mauritanie prévoit l'égalité des droits des citoyens en matière de vote et d'accès aux charges publiques et garantit la participation des femmes à la sphère publique et à la vie politique. En Mauritanie, 31 \% des postes ministériels et 25 \% des sièges parlementaires sont occupés par des femmes (UIP, 2017). En 2010, le Sénégal a adopté une loi instituant une parité femmes-hommes intégrale pour tous les organismes entièrement ou partiellement élus (CEDAW, 2014) ; en 2017, le pays s'est classé au 7e rang dans le monde pour la représentation des femmes au parlement (47\%).

\section{Graphique 2}

Moyenne SIGI en Afrique de l'Ouest : meilleure et plus faible performance
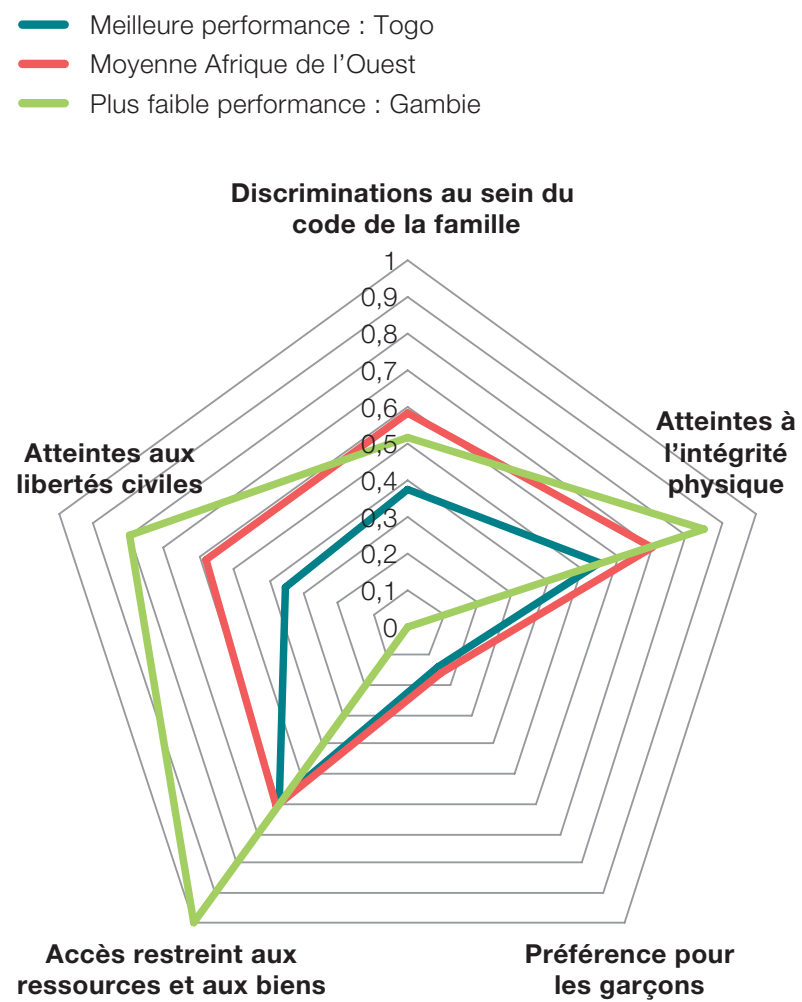

Notes : Ce graphique présente la moyenne régionale des scores dans les sous-indicateurs du SIGI ainsi que les scores du pays le plus performant (Togo) et du pays le moins performant (Gambie). Plus les valeurs sont élevées, plus le niveau de discrimination est élevé, l'indicateur allant de 0 (absence de discrimination) à 1 (discrimination très élevée).

Source : OCDE (2014a), Base de données Égalité homme-femme, Institutions et Développement, http://stats.oecd.org. 


\section{DISCRIMINATIONS AU SEIN DU CODE DE LA FAMILLE}

C e sous-indicateur aborde les institutions sociales déterminant le pouvoir décisionnel des femmes et leur statut au sein du ménage et de la famille. Le mariage, l'autorité parentale, les droits à l'héritage notamment, sont régis par des règles formelles et informelles, normes et pratiques sociales qui coexistent en droit civil ou droit commun, droit coutumier et lois religieuses.

Onze pays (Burkina Faso, Côte d'Ivoire, Gambie, Ghana, Libéria, Mali, Mauritanie, Niger, Nigéria, Sénégal et Tchad) présentent des niveaux élevés à très élevés, et aucun un niveau très faible ; ceci malgré le renforcement des cadres législatifs nationaux et des engagements régionaux.

\section{Mariage précoce}

Les pays ont ratifié des instruments juridiques contre la pratique du mariage précoce. Tous sont signataires du Protocole à la Charte africaine des droits de l'homme et des peuples relatif aux droits des femmes en Afrique, de la Charte africaine de la jeunesse (article 8) et de la Charte africaine des droits et du bien-être de l'enfant (article 21). Parmi les changements notables apportés aux cadres législatifs nationaux figure l'adoption par la Gambie d'un amendement à la loi sur l'enfance (2016), qui criminalise le mariage et les fiançailles d'enfants, et prévoit une peine de 20 ans de prison pour les personnes reconnues coupables de ces crimes (Département d’État des États-Unis, 2017).

Certaines lacunes juridiques et pratiques coutumières discriminatoires affaiblissent les droits des femmes. Dans 11 pays, les filles peuvent encore se marier avant l'âge de 18 ans (OCDE, 2014a)7. Même lorsque des lois interdisent le mariage des enfants, elles peuvent être affaiblies par la pluralité des systèmes juridiques. Ainsi, alors que le code civil peut fixer l'âge légal du mariage à 18 ans, d’autres régimes juridiques - droit coutumier, lois religieuses - peuvent permettre que les mariages précoces perdurent dans certains pays (comme en Gambie, au Ghana, en Mauritanie ou au Nigéria).

L'existence de campagnes et de plans d'action nationaux témoignent d'une volonté politique croissante dans certains pays. Le Ghana a mené une campagne nationale et créé en 2016 un Groupe de coordination sur le mariage d'enfants au sein du ministère du Genre, de l'enfance et de la protection sociale. Un cadre stratégique national de 10 ans a été formulé et une campagne, Mettre fin aux mariages d'enfants, lancée. En 2015, avec l'aide des Nations Unies et des organisations de la société civile, le ministère de la Femme, de la solidarité nationale et de la famille du Burkina Faso a établi une Feuille de route de lutte contre le mariage des enfants et les mutilations génitales féminines (2016-18). Cette feuille de route a quatre objectifs : 1) coordination, suivi et contrôle ; 2) mobilisation sociale et plaidoyer ; 3) renforcement des capacités des acteurs ; et 4) réponses multisectorielles (MFASSN, 2015).

Bien qu'elle ait légèrement diminué, la prévalence de cette pratique reste une préoccupation majeure. Le taux de mariages précoces y est plus de deux fois supérieur à la moyenne mondiale (13 \%) : 30 \% des filles âgées de 15 à 19 ans sont mariées, divorcées, veuves ou liées par une union informelle. Sur les 20 pays où le taux de mariages précoces est le plus élevé dans le monde, 7 se trouvent en Afrique de l’Ouest (Burkina Faso, Guinée, Mali, Niger, Nigéria, Sierra Leone et Tchad), avec un taux atteignant $76 \%$ au Niger. Cependant, le pourcentage de femmes mariées avant l'âge de 18 ans a légèrement baissé dans la plupart des pays ces dernières années (Graphique 3). La baisse la plus notable a eu lieu en Gambie, avec une différence de 28 points de pourcentage entre les femmes âgées de 45 à 49 ans (58\% déclarent avoir été mariées avant l'âge de 18 ans) et les femmes âgées de 20 à 24 ans (30\%) en 2013. 
Graphique 3

La prévalence des mariages précoces reste élevée à travers les groupes d'âge en Afrique de l'Ouest

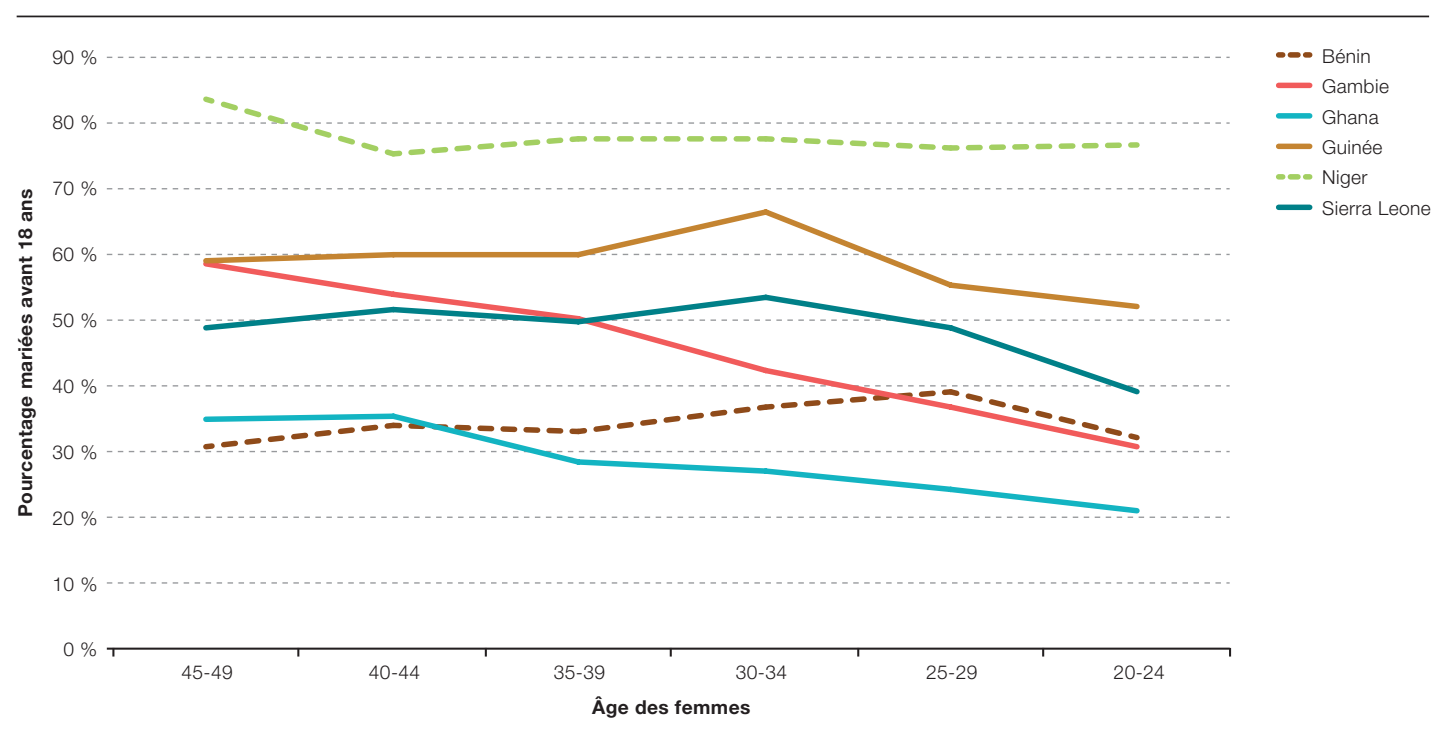

Note : Ce graphique présente le pourcentage de femmes mariées avant l'âge de 18 ans par groupe d'âge et par pays.

Source : Enquêtes démographiques et de santé (1988-2015), http://dhsprogram.com/data/.

Le mariage précoce est corrélé à des facteurs socio-économiques. Au Bénin, les filles vivant en milieu rural ont deux fois et demie plus de chances d'être mariées avant l'âge de 18 ans que les urbaines (Girls not Brides, n. d.). Au Sénégal, les filles des familles les plus pauvres sont quatre fois plus exposées au mariage précoce que celles des familles les plus riches (UNICEF, 2015) ; la pratique traditionnelle du mariage précoce est associée à un bénéfice social et financier pour la famille de la fille. La persistance de cette pratique s'appuie également sur une perception favorable qu'en a encore une partie de la population. Au Burkina Faso, par exemple, 44 \% de la population pense qu'il est acceptable qu'une fille se marie avant l'âge de 18 ans, contre seulement 4 \% pour un garçon (OCDE, 2018).

\section{Autorité parentale}

Une discrimination de jure continue de restreindre les droits des femmes en la matière. Seuls cinq pays (Bénin, Burkina Faso, Côte d'Ivoire, Gambie et Sierra Leone) reconnaissent l'égalité femmes-hommes pendant le mariage comme après un divorce. Des restrictions juridiques à l'autorité parentale des femmes, pendant et après le mariage, subsistent dans quatre pays (Guinée, Mali, Mauritanie et Tchad). De telles restrictions consistent, notamment, à reconnaître l'homme comme seul chef du ménage (Mali) ou à n'accorder l'autorité parentale qu'au père (Mauritanie).

Même lorsque la loi garantit l'égalité des droits, les femmes subissent souvent une discrimination de facto. Les lois garantissant l'égalité des droits sont souvent contredites par des pratiques coutumières, traditionnelles ou religieuses discriminatoires (Guinée-Bissau, Nigéria et Togo). Au Nigéria, dans le cadre des mariages civils, l'autorité parentale est partagée entre le père et la mère et, sur le plan juridique, les couples mariés sont conjointement responsables des dépenses de la famille. Les femmes, mariées ou non, peuvent être chefs de ménage au même titre que les hommes. Cependant, les deux tiers au moins de la population nigériane sont soumis à des lois coutumières et religieuses qui reconnaissent seulement à l’homme le statut de chef de ménage et l'autorité parentale. 
Encadré 1

Mariage précoce et éducation secondaire des filles en Afrique de l'Ouest

Dans les pays où la prévalence du mariage précoce est élevée, le taux de scolarisation au secondaire des filles est plus faible et elles finissent plus rarement leurs cursus (Centre de développement de l'OCDE, 2014) (graphique 4). Le mariage précoce est également associé à de moins bons résultats en matière de santé des filles et de leurs enfants, ainsi qu'à des taux de fécondité plus élevés. Les filles mariées de façon précoce sont plus susceptibles d'avoir un enfant à un jeune âge. Au Niger, 76 \% des femmes âgées de 20 à 24 ans étaient mariées avant l'âge de 18 ans et 48 \% avaient eu un enfant avant cet âge (UNICEF, 2015). De plus, l'acceptation sociale du mariage et de la grossesse précoces, l'accès limité à la contraception et la prévalence de la violence sexuelle favorisent des taux de fécondité plus élevés chez les adolescentes. La grossesse précoce écourte alors l'éducation des filles et explique les écarts persistants de scolarisation au secondaire entre filles et garçons.

\section{Graphique 4}

Plus la prévalence du mariage précoce est élevée, plus le taux de scolarisation des filles au secondaire est faible

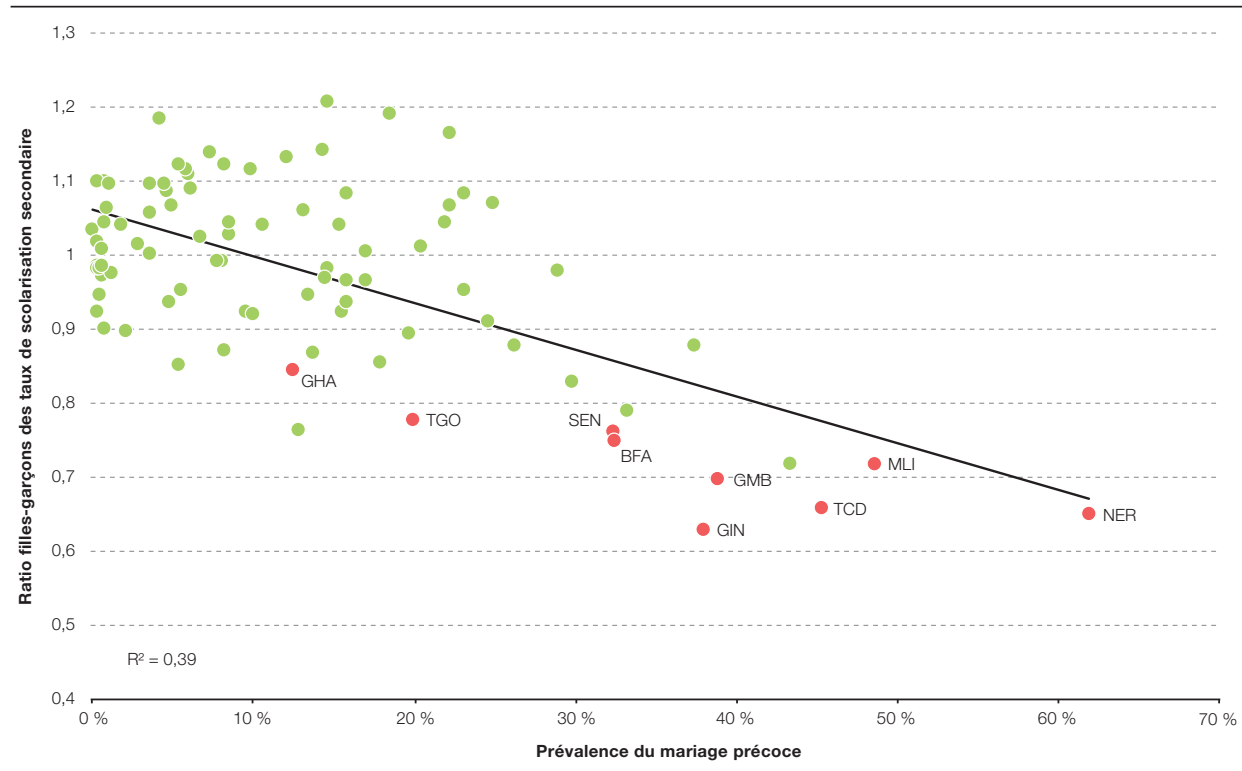

Note : Ce graphique illustre la relation entre le ratio filles-garçons des taux de scolarisation au secondaire et les niveaux de prévalence du mariage précoce des filles dans 88 pays, en tenant compte du PIB par habitant de chaque pays, de la proportion d'enseignants femmes, des dépenses d'éducation du gouvernement, de l'écart femmes-hommes en matière de chômage, du taux d'urbanisation et des caractéristiques régionales. $\mathrm{R}^{2}=0.39$ signifie que $39 \%$ des disparités régionales en termes de réussite des filles au secondaire s'expliquent par des différences régionales de prévalence du mariage précoce des filles.

Source : OCDE (2014a), Base de données Égalité homme-femme, Institutions et Développement, http://stats.oecd.org ; Banque mondiale (s.d.), Indicateurs du développement dans le monde (base de données), http://data.worldbank.org.

\section{Droits à I'héritage}

Dans la majorité des pays, les femmes sont victimes de discrimination lorsqu'elles demandent l'application de leurs droits. Les droits des veuves et des filles sont juridiquement protégés dans tous les pays, sauf cinq (Gambie, Mauritanie, Niger, Nigéria et Tchad). L'adoption au Togo, en 2012, du Code des personnes et de la famille, qui accorde l'égalité des droits aux veuves et aux filles, est une évolution positive. Néanmoins, dans certains pays, les lois coutumières et religieuses interdisent l'héritage aux femmes (Tchad) ; la propriété des biens revenant aux héritiers mâles (Guinée-Bissau). Au Mali, la portée du droit successoral est souvent limitée par l'idée que les femmes sont moins responsables et fiables que les hommes, et donc moins aptes à hériter des biens familiaux. En conséquence, les femmes héritent rarement de la terre quand l’héritage est divisé entre les ayants droit. 
La pratique du lévirat vise à préserver l'intégrité du patrimoine patrilinéaire et à s'assurer que la famille paternelle conserve la garde des enfants. Ce type de mariage, qui oblige la veuve à épouser le frère de son défunt mari, est courant en Afrique de l'Ouest et reste légal dans le cadre de certains droits coutumiers (Bénin, Mali). Dans d'autres communautés, lorsqu'une épouse meurt, sa jeune sœur est tenue d'épouser le veuf, pratique appelée sororat.

Dans sept pays de la région, seules $43 \%$ des veuves déclarent avoir hérité d'une partie des biens de leur défunt mari, et un plus petit nombre encore (27\%), de la majorité des biens. Dans six des sept pays étudiés (Bénin, Guinée, Mali, Niger, Nigéria et Sierra Leone), les enfants ou la famille du défunt héritent de la majorité des biens (Graphique 5). Les veuves ne sont susceptibles d'hériter de la majorité des biens qu'au Sénégal (46 \%).

\section{Graphique 5}

Qui hérite de la majorité des biens ? Pratiques en matière d'héritage dans quelques pays

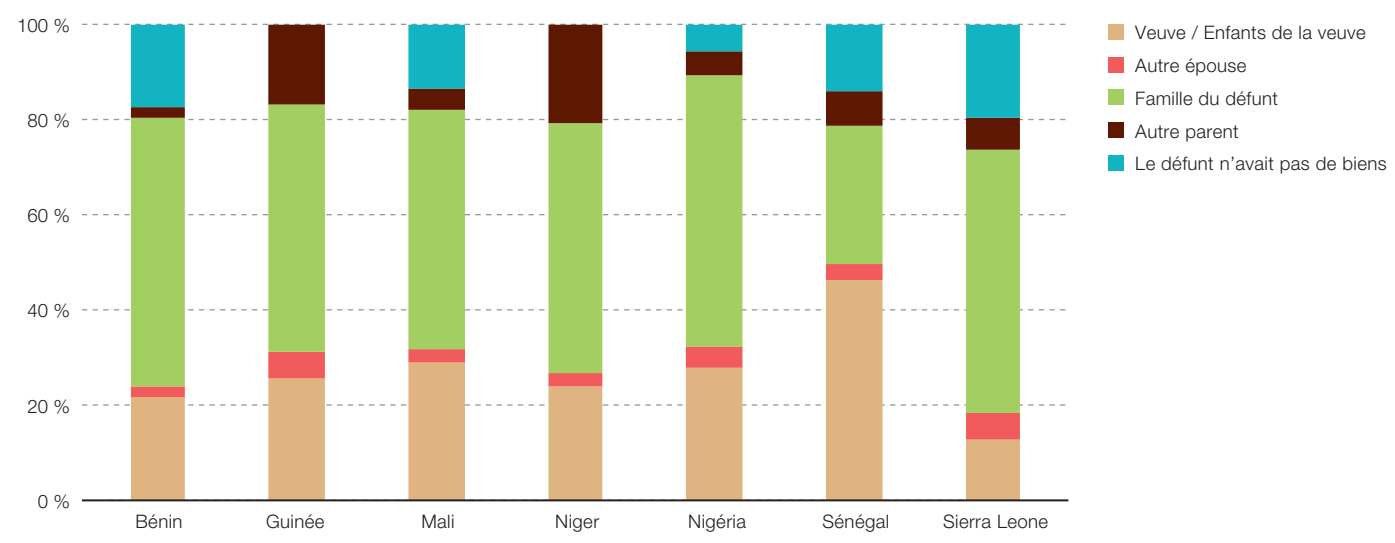

Notes : Ce graphique présente le pourcentage de cas dans lesquels la famille du défunt hérite de la majorité des biens, dans une sélection de sept pays. L'échantillon est tiré de la population de femmes âgées de 15 à 49 ans, et les valeurs moyennes sont calculées en utilisant les coefficients de pondération fournis dans les EDS.

Source : Enquêtes démographiques et de santé (1988-2015), http://dhsprogram.com/data.

\section{Divorce}

Dans la majorité des pays, la loi reconnaît aux femmes le droit d'entamer les procédures de divorce. Elle reste discriminatoire en Mauritanie et au Niger. Une femme mauritanienne ne peut demander le divorce qu'en invoquant un préjudice ou des blessures. Si elle a tort, elle doit verser une compensation (article 102). En revanche, le mari a le droit de la répudier (divorce par volonté unilatérale) sans avoir à donner de raison, ni à verser de compensation.

Néanmoins, dans huit pays (Gambie, Guinée, Libéria, Mali, Nigéria, Sénégal, Tchad et Togo), des coutumes ou des pratiques religieuses discriminatoires affectent la capacité des femmes à faire valoir leurs droits. Au Mali, si les deux époux peuvent demander le divorce, il est rare que les femmes entament les procédures, à cause de fortes pressions sociales et de la crainte de perdre la garde de leurs enfants. Au Togo, les femmes peuvent présenter une demande de divorce aux mêmes conditions que les hommes, et le nouveau Code des personnes et de la famille a introduit le divorce par consentement mutuel. Mais, dans les zones rurales où prédominent les mariages coutumiers, la répudiation constitue la forme la plus courante de divorce, et les femmes n'ont aucun droit à une pension alimentaire pour ex-conjoint ou pour enfant. Les femmes répudiées perdent la garde de leurs enfants et sont contraintes de retourner chez leurs parents en laissant derrière elles tous leurs biens. 


\section{ATTEINTES À L'INTÉGRITÉ PHYSIQUE}

C e sous-indicateur mesure les institutions sociales relatives au pouvoir des femmes et des filles sur leur corps, à la vulnérabilité des femmes et à la violence à leur encontre. Il couvre les lois formelles et informelles, les normes et les pratiques relatives à l'intégrité physique et à l'autonomie reproductive des femmes, la violence et les mutilations génitales féminines (MGF).

Quinze pays (Bénin, Burkina Faso, Côte d'Ivoire, Gambie, Ghana, Guinée, GuinéeBissau, Libéria, Mali, Mauritanie, Nigéria, Sénégal, Sierra Leone, Tchad et Togo) présentent des niveaux élevés à très élevés de discrimination. Si certains pays ont accru la protection légale des femmes, il reste des vides juridiques, en particulier en matière de violence domestique. Les niveaux d'acceptation sociale de la violence contre les femmes sont élevés, y compris parmi les femmes elles-mêmes. Les conflits augmentent la vulnérabilité des femmes à la violence fondée sur le sexe et limitent leur capacité à demander justice.

L'intégrité physique des femmes et des filles reste menacée par les MGF, malgré le renforcement des engagements régionaux. Le Protocole à la Charte africaine des droits de l'homme et des peuples relatif aux droits des femmes en Afrique, ou Protocole de Maputo (2003), appelle les États à adopter les mesures appropriées pour assurer la protection des femmes contre toutes les formes de violence et les pratiques néfastes, y compris les MGF. Il a été ratifié par 14 des 17 pays d'Afrique de l'Ouest; trois l'ayant signé mais non encore ratifié (Niger, Sierra Leone et Tchad). Néanmoins, l'élimination des MGF ne progresse que lentement.

\section{Violence domestique}

Des lacunes juridiques légitiment les pratiques coutumières qui accroissent la vulnérabilité physique des femmes. Huit pays (Burkina Faso, Côte d'Ivoire, Gambie, Libéria, Mali, Mauritanie, Niger et Tchad) n'ont pas de législation nationale. Par ailleurs, lorsqu'elles existent, les lois ne protègent que partiellement les femmes. Ainsi, le Sénégal exclut la violence sexuelle, émotionnelle et économique ; la Gambie et la Mauritanie ne reconnaissent pas le viol conjugal.

Graphique 6

Relation entre l'acceptation par les femmes de la violence domestique et le taux de prévalence (qu'il existe ou non un cadre juridique)

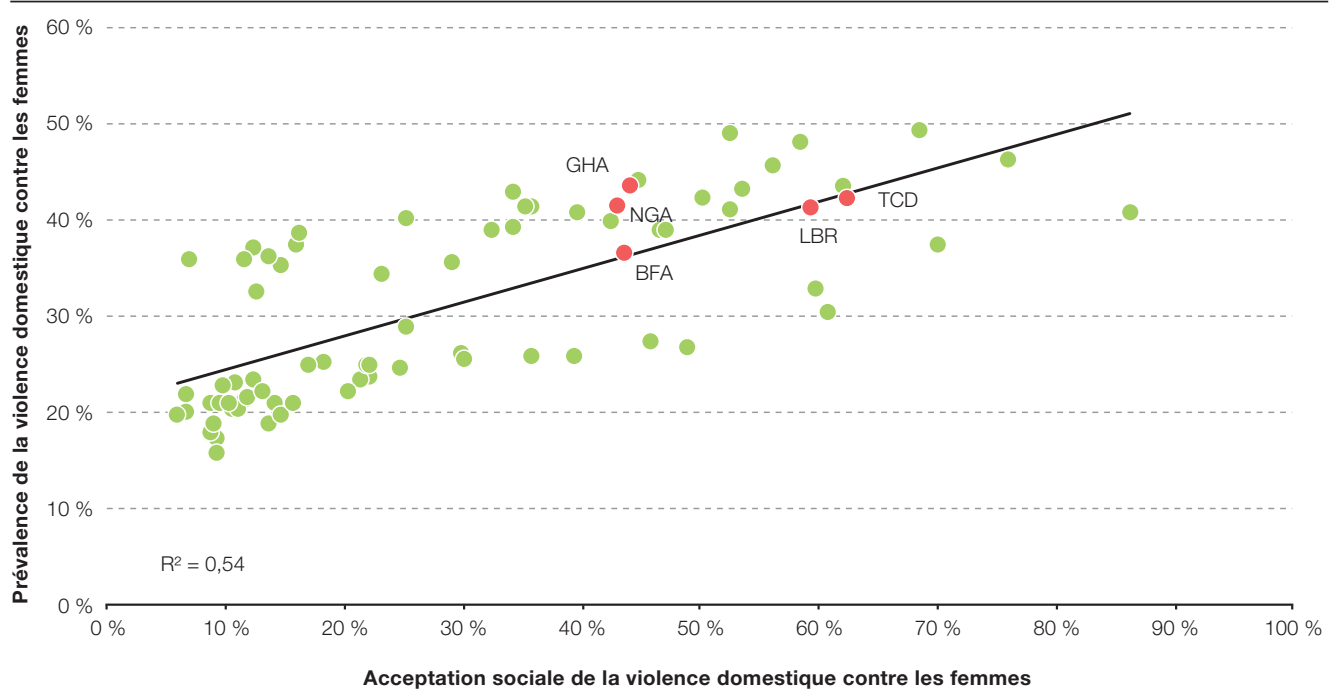

Note : Ce graphique présente la relation entre l'acceptation sociale de la violence domestique contre les femmes (mesurée par la part des femmes déclarant qu'il est justifié pour un mari de battre sa femme sous certaines conditions) et la prévalence de la violence domestique contre les femmes (mesurée par la part des femmes âgées de 15 à 49 ans déclarant avoir été victimes de violence physique et/ou sexuelle de la part d'un partenaire au cours de leur vie). Ces chiffres tiennent compte des caractéristiques de revenu et régionales, ainsi que du score SIGI sur l'existence de lois contre la violence domestique touchant les femmes. $\mathrm{R}^{2}=0.54$ signifie que $54 \%$ des disparités nationales en termes de prévalence de la violence domestique s'expliquent par des différences d'acceptation de la violence domestique.

Source : OCDE (2014a), Base de données Égalité homme-femme, Institutions et Développement, http://stats.oecd.org. 
La violence domestique reste courante et largement justifiée par les normes sociales. En moyenne au Nigéria, 18 \% des femmes ont été victimes de violence physique ou sexuelle de la part d'un partenaire au cours de leur vie, proportion qui atteint $57 \%$ des femmes en Sierra Leone (OCDE, 2014a). Cinquante-sept pour cent des femmes pensent qu'il est justifié sous certaines conditions que leur mari ou leur partenaire les batte, proportion qui atteint 92 \% en Guinée. Les normes sociales justifiant la violence domestique contribuent à expliquer ses taux de prévalence élevés, même dans les pays dotés d’une législation appropriée : une prévalence plus élevée correspond à des niveaux d'acceptation sociale plus élevés. Au Nigéria, 18 \% des femmes déclarent avoir subi de la violence de la part d'un partenaire intime au cours de leur vie, et $43 \%$ acceptent cette pratique, tandis que les chiffres sont nettement plus élevés au Tchad, avec $57 \%$ de femmes victimes de violence, et $62 \%$ acceptant cette pratique (Graphique 6).

\section{Violence sexuelle}

Des lacunes importantes subsistent dans les législations. Des lois sur le viol existent dans la majorité des pays. Elles ne sont complètes qu'au Bénin, Burkina Faso et Ghana. Dans ces pays, le viol conjugal est couvert et les poursuites contre l'auteur des actes ne sont pas abandonnées s'il épouse sa victime. Même dans ces trois pays, l'application de la loi est inefficace. Dans les 14 pays restants, des lacunes juridiques dans les lois sur le viol profitent aux auteurs des actes. Par exemple, le viol conjugal n'est pas couvert dans 10 pays.

Les lois sont mal appliquées et les programmes manquent de ressources. En Côte d'Ivoire, la Stratégie nationale de lutte contre les violences basées sur le genre a créé un budget spécial d’application (MSFFE, 2014). Ceci est une exception. Généralement, même lorsque les auteurs des faits sont arrêtés, il arrive qu'aucune enquête ne soit menée ou que les poursuites soient abandonnées avant que la justice ait rendu un verdict (Département d'État des États-Unis, 2012). Assurer une formation spécialisée aux officiers de police, ainsi qu'aux professionnels de la justice et de la santé, fait partie de la solution. Au Nigéria, ONU Femmes a organisé des ateliers de formation destinés aux officiers de police et contribué à l'élaboration d'une Politique interne sur l'égalité des sexes adaptée au contexte de la police nationale (2009). En Guinée, le gouvernement a lancé un programme de renforcement des capacités en matière de soins médicaux et de services de conseil destiné aux professionnels de la santé et mis en place un service d’assistance téléphonique pour les victimes de viol et de violence sexuelle (MASPFE, 2014). Les gouvernements de la région ont reconnu la nécessité d'une approche holistique pour répondre aux besoins spécifiques des victimes de violence.

La stigmatisation des femmes et la négation de leurs droits renforcent les institutions sociales discriminatoires. Au Mali, 78 \% des femmes âgées de 15 à 49 ans déclarent qu'il n'est pas justifié de refuser d'avoir des relations sexuelles avec son mari, contre $5 \%$ au Tchad. La stigmatisation au sein du système judiciaire peut dissuader les femmes de faire valoir leurs droits. Au Nigéria et au Sénégal, la police ne veut pas intervenir dans les cas de violence domestique, car celle-ci est perçue comme une affaire d'ordre privé (OCDE, 2014b).

Les conflits aggravent la vulnérabilité des femmes. En Côte d'Ivoire, durant la crise de 2001, 67 \% des femmes ont été victimes de violence fondée sur le sexe (BAD, 2013). En 2007, la Côte d'Ivoire a été le premier pays sortant d'un conflit à adopter un Plan national d'action pour la mise en œuvre de la Résolution 1325 du Conseil de sécurité des Nations Unies sur les femmes, la paix et la sécurité (RCSNU 1325), suivie par le Libéria en 2009. Au Libéria, un service spécialisé du ministère de la Justice est chargé des cas de violence sexuelle et de violence fondée sur le sexe (ONU Femmes, 2015).

Les femmes ne bénéficient pas d'une protection juridique complète contre le harcèlement sexuel. Dans près de la moitié des pays (8 sur 17), des lois couvrent une forme ou une autre de harcèlement sexuel, mais la plupart ne protègent les femmes que dans des domaines limités. Dans cinq pays (Burkina Faso, Côte d’Ivoire, Niger, Sénégal et Togo), 
Encadré 2

Mortalité maternelle et « femmes manquantes » en Afrique de l'Ouest

Dans d'autres régions (Asie de l'Est et Pacifique, Asie centrale et Caucase, et Asie du Sud, notamment), le phénomène des « femmes manquantes » (proportion trop faible des femmes dans la population) est la conséquence des avortements sélectifs et des infanticides selon le sexe. En Afrique de l'Ouest, les ratios hommes-femmes à la naissance correspondent aux tendances naturelles et ne mettent donc pas en évidence de préférence pour les garçons (graphique 7). En revanche, la mortalité des femmes est alarmante dans la cohorte des 15-60 ans, en particulier chez les 15-49 ans.

Graphique 7

Nombre d'hommes pour 100 femmes par groupe d'âge

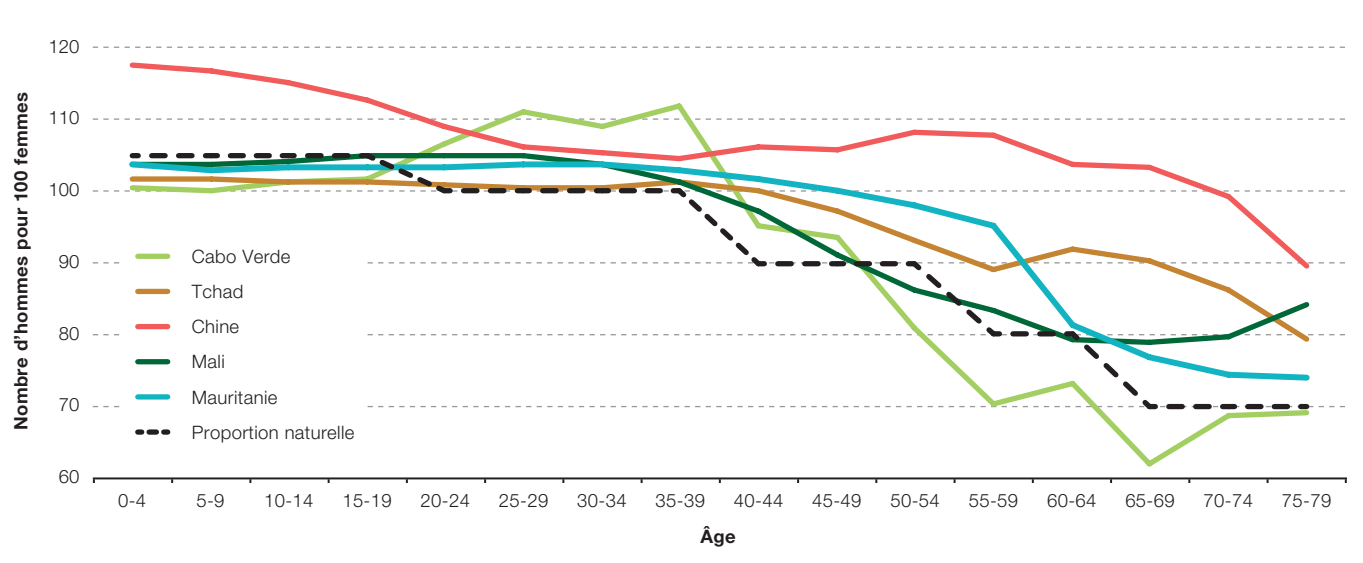

Note : Ce graphique présente le nombre d'hommes pour 100 femmes (sex ratio), par groupe d'âge. Source : Nations Unies (2015c).

Ceci s'explique par des taux élevés de mortalité maternelle. Ces taux ont diminué - 1132 décès pour 100000 naissances vivantes en 1990 ; 678 décès en 2015 (Banque mondiale, s.d.) - mais demeurent plus de trois fois supérieurs à la moyenne mondiale qui est de 216 (Banque mondiale, s.d.). C'est en Sierra Leone et au Tchad que les femmes ont le plus de risques de mourir de complications liées à la grossesse, les taux de mortalité maternelle y atteignant respectivement 1 pour 17 et 1 pour 18 , contre une moyenne mondiale de 1 pour 180 (Banque mondiale, s.d.). Le Cabo Verde est le seul pays où le risque de mortalité maternelle est inférieur à la moyenne mondiale (1 pour 900).

seul est concerné le harcèlement sexuel au travail. Seuls quatre pays (Bénin, Cabo Verde, Gambie et Nigéria) s'attaquent au harcèlement sexuel en milieu scolaire, et deux (Bénin et Guinée-Bissau) le font dans l'espace public (Banque mondiale, 2015).

\section{Mutilations génitales féminines}

Si le nombre de cadres juridiques nationaux a augmenté, leur portée et leurs mécanismes de mise en œuvre varient. La pratique des MGF est criminalisée dans tous les pays sauf quatre (Cabo Verde - où les MGF n'ont aucun ancrage culturel -, Libéria, Mali et Sierra Leone). Cependant, le décret adopté en Mauritanie (2005) interdit seulement qu'elles soient pratiquées sur des mineures dans des établissements médicaux. De plus, les lois sont souvent peu appliquées : au Burkina Faso, où elles sont interdites depuis 1996, seules sept condamnations ont été prononcées en 2012 (UNICEF, 2013).

Malgré des signes positifs indiquant un recul des MGF, les taux restent élevés, notamment en raison de l'acceptation sociale de cette pratique. Plus de $46 \%$ des femmes ouest-africaines ont été victimes de MGF ; neuf femmes sur dix en Guinée et au Mali. Dans ces deux pays, l'acceptation par les femmes elles-mêmes reste élevée : 76 \% en Guinée, 72 \% au Mali (UNICEF, 2013). Cette pratique est souvent perçue comme un rite de passage à l'âge adulte et un facteur déterminant de leur (c mariabilité ») ; la décision est souvent prise sous la pression des familles élargies et des communautés, et rarement 
avec le consentement de la victime (UNICEF, 2013). Néanmoins, le soutien aux MGF semble diminuer, en particulier au sein des jeunes générations. En Sierra Leone, 98 \% des femmes âgées de 45 à 49 ans ont été excisées, contre $74 \%$ des filles âgées de 15 à 19 ans. Ces dernières sont $57 \%$ à penser que la pratique devrait perdurer, contre $81 \%$ de leurs aînées (DHS, 2013).

\section{Autonomie reproductive}

Les femmes ont une faible autonomie reproductive. Une femme sur quatre a un besoin en planification familiale non satisfait, soit deux fois plus que la moyenne mondiale. En outre, le recours à la contraception est le plus faible (17\%, contre $64 \%$ à l'échelle mondiale) (NU, 2015b).

La faible autonomie reproductive des femmes est liée à la disponibilité insuffisante de services de santé dans la région. Les difficultés d’accès aux services de santé reproductive sont plus grandes encore pour les femmes pauvres et les femmes vivant en milieu rural, la pauvreté et l'éloignement géographique affectant leurs ressources déjà limitées (UNFPA, 2012).

\section{Encadré 3}

Burkina Faso : Les institutions sociales discriminatoires contribuent à des taux de fécondité élevés

Les femmes ont généralement plus d'enfants lorsqu'elles sont confinées à leur rôle reproductif et n'ont qu'une autonomie reproductive limitée, notamment un faible pouvoir décisionnel sur l'utilisation des contraceptifs, le nombre d'enfants et l'espacement des naissances, et un accès limité à la planification familiale. Au Burkina Faso, les données montrent des corrélations entre les régions où les taux de fécondité sont élevés et la prévalence d'opinions et de pratiques discriminatoires (graphique 8). L'acceptation et la prévalence du mariage et des grossesses précoces sont liées à des niveaux d'éducation des filles plus faibles et à des taux de fécondité plus élevés.

Graphique 8

Plus les niveaux de discrimination dans les institutions sociales sont élevés, plus les taux de fécondité sont élevés

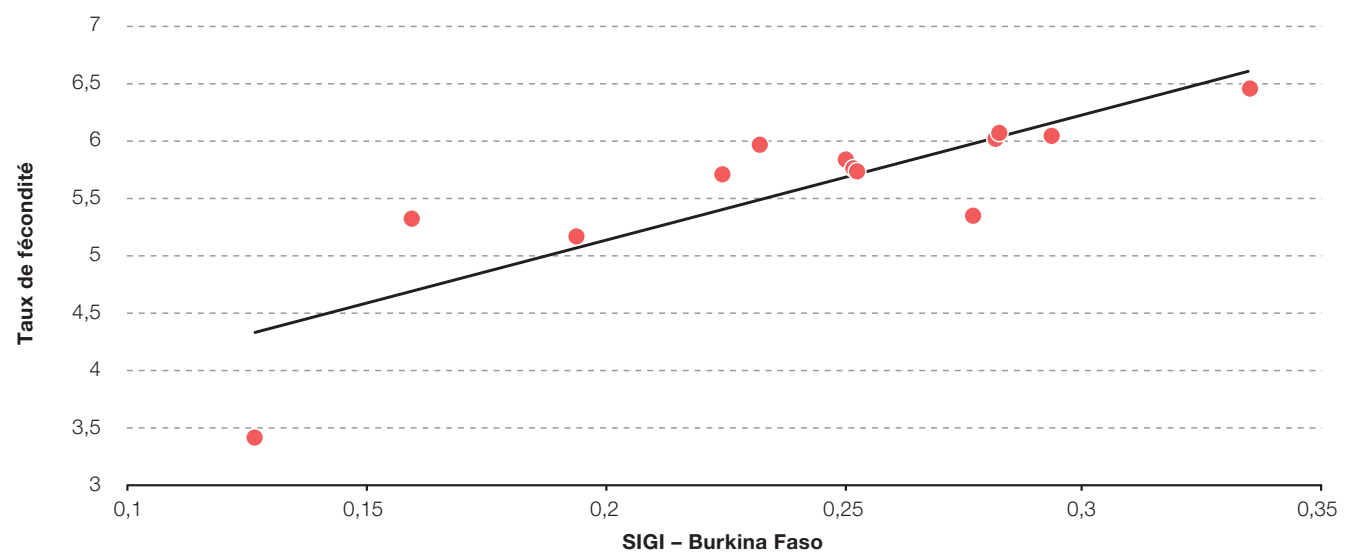

Note : Ce graphique présente la relation entre la discrimination à l'encontre des femmes au sein des institutions sociales (mesurée par le SIGI-Burkina Faso) et les taux de fécondité (nombre moyen d'enfants par femme âgée de 15 à 49 ans) au niveau régional, avec pour variables de contrôle le niveau d'urbanisation, d'éducation et de revenu, le taux d'emploi des femmes, l'âge, la taille du ménage et la part de la population de religion catholique et musulmane.

Source : EDS (2014), OCDE (2018). 


\section{ACCÈS RESTREINT AUX RESSOURCES ET AUX BIENS}

e sous-indicateur considère les discriminations en matière d'accès aux ressources naturelles et économiques et de pouvoir décisionnel sur celles-ci.

Douze pays (Bénin, Burkina Faso, Côte d'Ivoire, Gambie, Ghana, Guinée-Bissau, Mauritanie, Niger, Nigéria, Sierra Leone, Tchad et Togo) affichent des taux de discrimination élevés à très élevés en la matière. Des pratiques coutumières discriminatoires et la mauvaise application des cadres législatifs expliquent d'importants écarts femmes-hommes en matière de propriété foncière et non foncière. Les veuves sont particulièrement vulnérables à la dépossession des biens et au déshéritage.

L'égalité en matière de propriété foncière et non foncière et d'accès aux services financiers est porteuse de nombreux effets positifs sur la nutrition, l'éducation, la sécurité alimentaire, la productivité agricole et le bien-être des femmes (van den Bold et al., 2015 ; Jones et al., 2010). Inversement, la faiblesse des taux de propriété des femmes et de leur pouvoir décisionnel sur les terres et les biens est corrélée à une vulnérabilité accrue des femmes et de leur famille, à la pauvreté et à moins d'opportunités d'activités génératrices de revenus pour les femmes (FAO, 2011 ; Centre de développement de l'OCDE, 2014).

\section{Accès sécurisé à la terre}

L'égalité juridique, reconnue dans la plupart des constitutions et lois nationales, se heurte à des attitudes et pratiques discriminatoires profondément ancrées. Dans tous les pays, le droit coutumier empêche les femmes d'accéder à la terre en bloquant leurs droits à en hériter ou à en posséder en leur nom propre. Au Libéria, si le droit foncier et la constitution reconnaissent aux femmes et aux hommes les mêmes droits, le droit coutumier, qui favorise la propriété des hommes, tend à prévaloir. Au Bénin, si le Code foncier rural (2007) reconnaît l'égalité des droits en matière de propriété de la terre, le droit coutumier stipule que seuls les hommes peuvent hériter de la terre. En Sierra Leone, le droit coutumier s'applique dans 12 districts sur 14, bien que la constitution reconnaisse aux femmes mariées et non mariées les mêmes droits qu'aux hommes en matière de propriété foncière.

Pourtant, les femmes contribuent significativement à la production agricole et alimentaire en tant que productrices, travailleuses agricoles familiales et transformatrices des aliments. Au Burkina Faso, au Cabo Verde, en Côte d'Ivoire, en Gambie, en Guinée, au Mali et au Sénégal (Graphique 9), les femmes représentent en moyenne $43 \%$ des travailleurs agricoles, mais seulement $8 \%$ des propriétaires de parcelles agricoles.

\section{Graphique 9}

Part des femmes parmi les propriétaires et les travailleurs agricoles

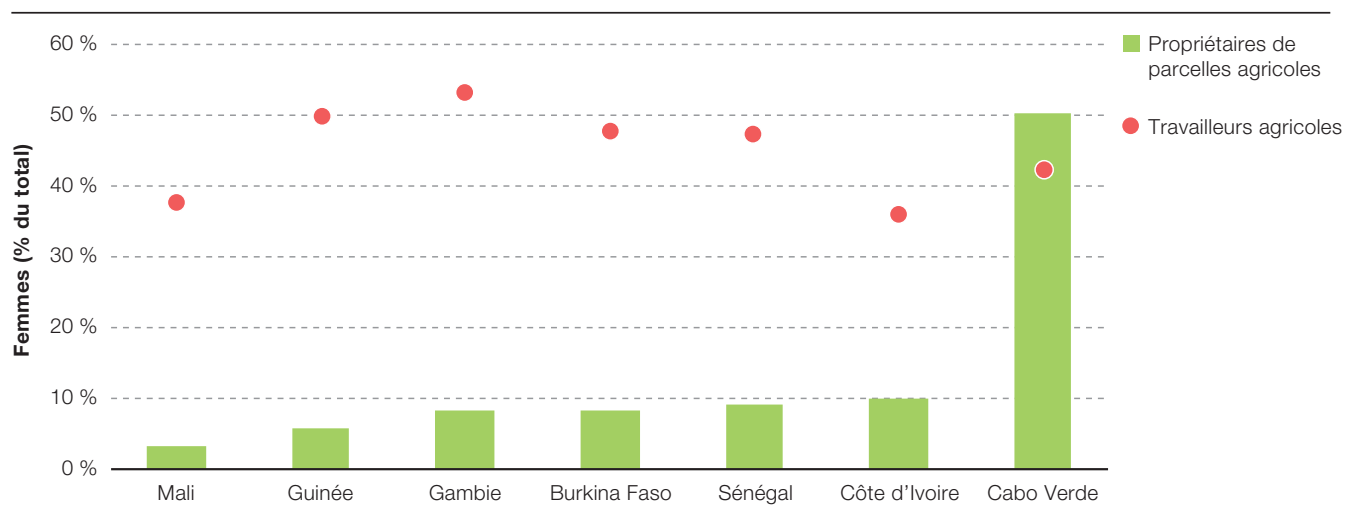

Note : Ce graphique présente la part des femmes parmi les propriétaires de parcelles agricoles et les travailleurs agricoles en pourcentage du total de ces propriétaires et travailleurs, par pays.

Source : FAO (2011). 
Encadré 4

Travail non rémunéré et participation des femmes au marché du travail

Les femmes portent le fardeau des activités non rémunérées. Elles consacrent en moyenne six fois plus de temps que les hommes au travail domestique et de soin non rémunéré ; cependant, ce ratio varie beaucoup selon les pays, de 1 à 2 au Nigéria à 1 à 17 au Mali. Cette division inégale résulte d'institutions sociales discriminatoires au niveau des ménages, les femmes étant perçues comme responsables de ces tâches. Le manque d'infrastructures contraint également les familles à consacrer plus de temps à la collecte d'eau et de combustible pour cuisiner ; cela affecte de façon disproportionnée les femmes et les filles, qui assument $71 \%$ des activités de collecte d'eau en Afrique subsaharienne (NU, 2015a).

Il existe une forte corrélation négative entre le temps consacré au travail de soin non rémunéré et les taux de participation des femmes au marché du travail (graphique 10). Ces restrictions ont d'importantes conséquences macro-économiques, la perte de revenu attribuée aux inégalités femmes-hommes sur le marché du travail atteignant un niveau substantiel, de 1 \% du PIB au Ghana et au Libéria à 31 \% au Nigéria (Cuberes et Teignier, 2013).

\section{Graphique 10}

Les inégalités en matière de travail domestique et de soin non rémunéré sont corrélées aux inégalités sur le marché du travail

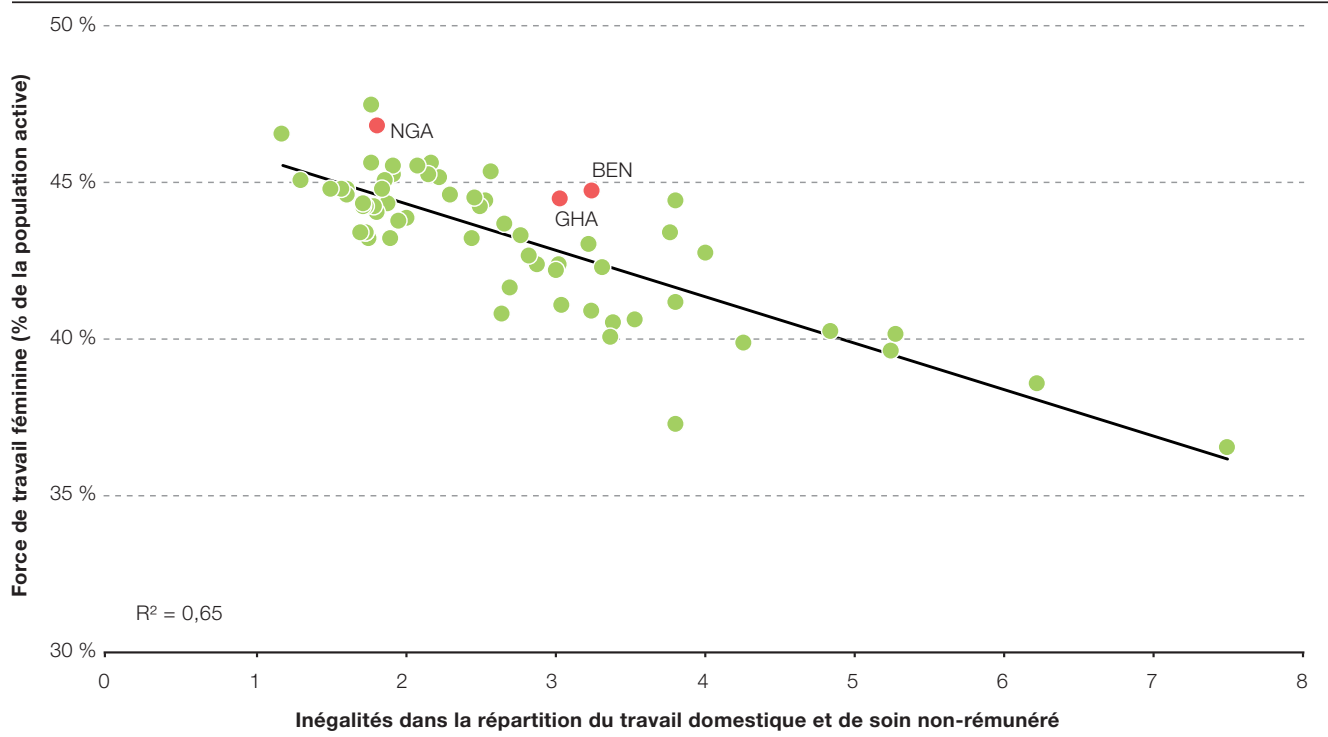

Note : Ce graphique présente la part estimée des femmes dans la population active (en pourcentage de la population active totale) en fonction des niveaux d'inégalité femmes-hommes en matière de travail de soin non rémunéré (mesurés par le ratio femmes-hommes du temps consacré aux activités domestiques et de soin non rémunérées), en prenant en compte le PIB par habitant, le taux de fécondité, le taux d'urbanisation, les congés de maternité, les inégalités femmes-hommes en termes d'emploi et d'éducation, et les caractéristiques régionales. $\mathrm{R}^{2}=0.65$ signifie que $65 \%$ des disparités nationales en termes de participation des femmes à la population active s'expliquent par des inégalités femmes-hommes-en matière de travail domestique et de soin non rémunéré.

Source : Ferrant, Pesando et Nowacka (2014)

Le poids du travail domestique et de soin non rémunéré affecte leur capacité à consacrer du temps aux activités agricoles (Banque mondiale, 2017). Au Niger, une corrélation positive a été démontrée entre le nombre d'enfants dans le ménage et l'importance de l'écart de productivité agricole hommes-femmes au détriment de ces dernières. À l'inverse, au Nigéria, la production agricole des hommes augmente avec l'accroissement du nombre d'enfants dans le ménage, peut-être en raison de leur capacité à mobiliser les membres du ménage les plus jeunes pour travailler sur le terrain. Cette inégalité peut également être le reflet de l'importance respective accordée aux parcelles des hommes et à celles des femmes (Banque mondiale, 2014a). Conséquence de ces obstacles à la participation économique des femmes et à leur accès à la terre, celles-ci ont tendance à s'orienter vers les segments situés en aval des chaînes de valeur agroalimentaires (transformation, commercialisation, restauration), où elles représentent entre $72 \%$ et 88 \% des employés (Allen, Heinrigs et Heo, 2018, à paraître). 


\section{Accès sécurisé aux biens non fonciers}

Si la loi garantit dans la plupart des pays les droits des femmes de posséder et de gérer des biens non fonciers, les lacunes juridiques et les pratiques discriminatoires restent courantes. Au Togo et en Côte d'Ivoire, les femmes mariées peuvent posséder des biens fonciers et non fonciers de façon indépendante ; cependant, sous le régime de la communauté de biens, l'époux est reconnu comme le seul administrateur légal des biens de la famille. En réalité, au Togo, seules 10 \% des femmes sont propriétaires de leur logement, contre 30 \% des hommes (DHS, 2014). Leur manque de connaissances juridiques constitue également un obstacle empêchant les femmes de faire valoir leurs droits ; ainsi, même lorsqu'il existe une protection juridique, les femmes peuvent ne pas en être conscientes (comme au Mali) (OCDE, 2014a).

\section{Accès aux services financiers}

Les discriminations de droit et/ou de fait sont importantes dans plus des deux tiers des pays. Les banques exigent souvent des garanties que les femmes peuvent rarement apporter, ou la signature du mari. L'analphabétisme et le manque de connaissances des affaires compliquent également l'accès des femmes au crédit (Libéria). En 2014, 23 \% des femmes ouest-africaines avaient un compte dans une institution financière, contre $36 \%$ des hommes (Banque mondiale, 2014b) ${ }^{8}$. Faciliter l'accès des femmes à la microfinance est une stratégie courante pour surmonter cette discrimination.

Les inégalités femmes-hommes concernant la propriété de la terre et des biens aggravent les difficultés (Graphique 11). Faute de posséder de la terre ou des biens, les femmes ont moins de chances d'obtenir un prêt bancaire en raison de l'exigence de garantie (O’Sullivan et al., 2014 ; FAO, 2014). Comme le confirment les résultats de l’Indice d'autonomisation des femmes dans l'agriculture, « le premier facteur contribuant à la désautonomisation des femmes réside dans l'accès au crédit et les décisions touchant le crédit ») (Alkire et al., 2013). Cela a de vastes implications pour l'autonomisation économique des femmes, notamment en termes de capacité à agrandir ou améliorer les parcelles familiales, à se doter de nouvelles technologies, ou à créer ou faire croître une entreprise (O’Sullivan et al., 2014 ; FAO, 2014).

Graphique 11

Au Burkina Faso, les restrictions à l'accès des femmes à la terre aggravent les restrictions à l'accès des femmes aux services financiers

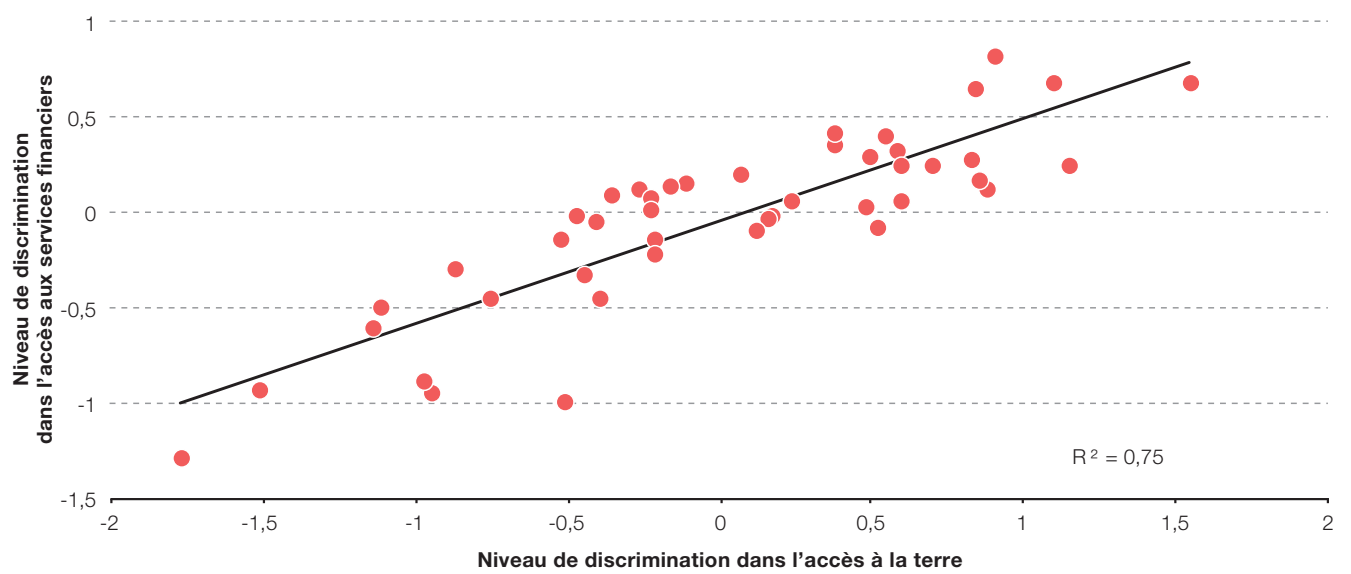

Note : Ce graphique présente la relation entre le niveau de discrimination à l'encontre des femmes dans l'accès à la terre au Burkina Faso et la valeur prédite du niveau de discrimination dans l'accès aux services financiers au niveau provincial, en prenant en compte le niveau de pauvreté, d'urbanisation et d'éducation au niveau provincial. Une valeur de -1.5 indique un niveau minimal de discrimination, et une valeur de 1.5 un niveau maximal de discrimination. $\mathrm{R}^{2}=0.75$ signifie que $75 \%$ des disparités entre provinces en termes d'accès des femmes aux services financiers s'expliquent par des différences de discrimination en termes d'accès à la terre.

Source : OCDE (2018), Étude pays SIGI-Burkina Faso, http://stats.oecd.org. 
Encadré 5

Le coût de la discrimination en matière de croissance économique et de bien-être

Les institutions sociales discriminatoires affectent la croissance. Le niveau actuel de discrimination de genre au sein des institutions sociales entraîne une perte de revenu de 12000 milliards de dollars (USD), soit $16 \%$ du revenu mondial. Pour les pays d'Afrique de l'Ouest, la perte totale atteint 120 milliards de dollars (USD) (Ferrant et Kolev, 2016).

Elles nuisent également au bien-être. Les hommes comme les femmes sont plus heureux quand ils vivent dans un pays qui leur garantit l'égalité en matière de droits et d'opportunités, indépendamment des caractéristiques du pays et des individus (Ferrant et al., 2017).

\section{ATTEINTES AUX LIBERTÉS CIVILES}

C e sous-indicateur traite des lois et pratiques relatives à l'accès des femmes à l'espace public, leurs libertés civiles et leur participation à la vie politique ; y compris la liberté de mouvement, l'exercice du droit citoyen (vote en particulier), la latitude à briguer une charge publique, les comportements envers les dirigeantes ou personnalités publiques.

Onze pays (Bénin, Côte d'Ivoire, Gambie, Ghana, Guinée-Bissau, Libéria, Mali, Niger, Nigéria, Sierra Leone et Tchad) présentent des niveaux d'atteintes aux libertés civiles élevés à très élevés. Cinq pays (Burkina Faso, Guinée, Mauritanie, Sénégal et Togo) présentent des niveaux de discrimination faibles à intermédiaires, et aucun pays ne présente un niveau très faible.

Les quotas imposés par les gouvernements ont beaucoup contribué à accroître la représentation politique des femmes; souvent sous la pression d'organisations de la société civile. Par ailleurs, le Protocole à la Charte africaine des droits de l'homme et des peuples relatif aux droits des femmes en Afrique (2003) soutient les mesures prises aux niveaux national et local pour accroître la représentation politique des femmes. Dans l'Agenda 2063 de l'Union africaine, les gouvernements soulignent l'importance de briser le ( plafond de verre politique » qui entrave la progression des femmes et empêche une parité femmes-hommes complète aux postes d'élus politiques et aux postes à responsabilités au sein du gouvernement et du secteur privé.

Cependant, la discrimination contre les femmes perdure dans la sphère publique, notamment sous la forme d'attitudes patriarcales qui justifient la violence contre les femmes. Ceci continue à priver les femmes d'un accès libre et sécurisé à l'espace public et à restreindre leur participation politique.

\section{Accès à l'espace public}

Si beaucoup d'obstacles juridiques sont levés, des pratiques discriminatoires ou des lois informelles restreignent l'accès libre et sécurisé des femmes aux lieux publics alors qu'elles jouissent de ces droits. C'est le cas au Bénin, au Burkina Faso, en Gambie, en Guinée, au Libéria, au Mali, au Nigéria, au Sénégal, en Sierra Leone et au Tchad. Au Burkina Faso, 91 \% de la population estime que les femmes doivent demander l'autorisation de leur mari avant de sortir de la maison (OCDE, 2018).

\section{Participation à la vie politique}

Six pays (Burkina Faso, Cabo Verde, Guinée, Mauritanie, Niger et Sénégal) ont mis en place aux niveaux national et local des quotas pour promouvoir la participation politique des femmes ; la Sierra Leone a adopté des quotas électoraux au seul niveau national. Si la représentation des femmes au parlement s'est accrue dans la région, passant de $13 \%$ en 2007 à près de $16 \%$ en 2017 (UIP, 2017) ${ }^{9}$, il reste de fortes disparités entre les pays ; de $43 \%$ au Sénégal à $6 \%$ au Nigéria (UIP, 2017) (Graphique 12). 
Graphique 12

Représentation politique des femmes et quotas

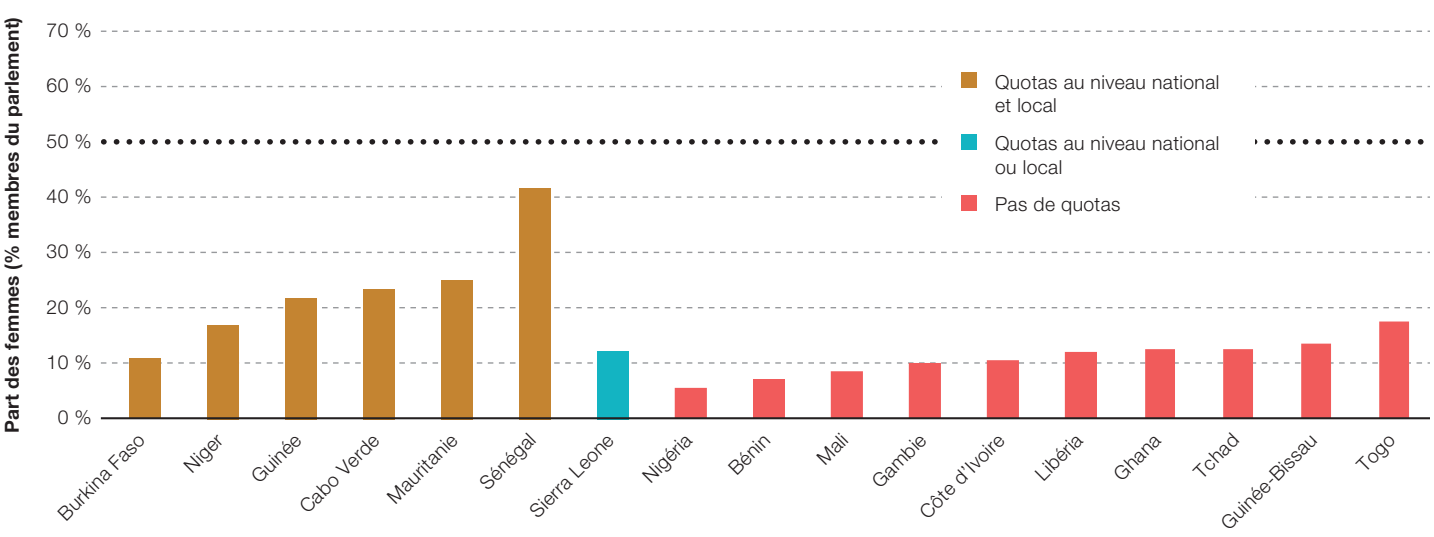

Note : Ce graphique présente la part des femmes en pourcentage de sièges au parlement selon le type quotas en place dans le pays.

Source : OCDE (2014a), Base de données Égalité homme-femme, Institutions et Développement, http://stats.oecd.org.

\section{Encadré 6}

Participation politique des femmes et gouvernance

Si la présence des femmes en politique et dans les structures décisionnelles gouvernementales favorise les progrès en matière de gouvernance démocratique, d'éducation, d'infrastructures ou de normes sanitaires, leur accès aux postes de dirigeants politiques reste un défi. Les attitudes négatives à l'égard des femmes en tant que leaders politiques et communautaires sont associées à une moindre participation des femmes à la politique (Graphique 13, partie gauche). Pourtant, les pays dont le parlement compte une proportion de femmes plus importante bénéficient d'une meilleure gouvernance (Graphique 13, partie droite).

Graphique 13

Attitudes, participation politique des femmes et gouvernance

\section{$50 \%$}

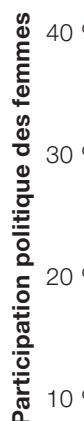

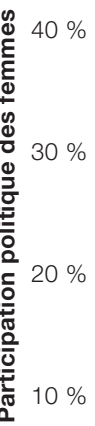

$$
0 \% \stackrel{R^{2}=0.46}{0 \%}
$$

Attitudes défavorables à la participation politique des femmes

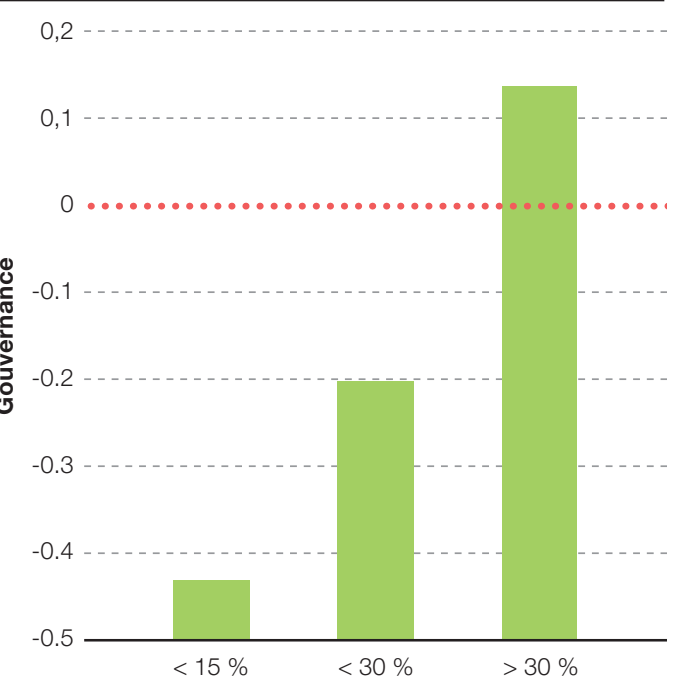

Participation politique des femmes

Notes : La partie gauche du graphique présente la relation entre les attitudes à l'égard des femmes en tant que dirigeantes politiques (pourcentage de la population qui pense que les hommes sont de meilleurs dirigeants politiques que les femmes) et la proportion de femmes au sein du parlement national de 100 pays en 2014 , en prenant en compte l'existence de quotas, les caractéristiques régionales et le niveau de revenu du pays. $R^{2}=0.46$ signifie que $46 \%$ des disparités entre pays en termes de participation politique des femmes s'expliquent par des différences régionales en termes d'attitudes à l'égard des femmes en tant que leaders politiques, de caractéristiques régionales et de revenu. La partie droite du graphique présente le niveau de gouvernance des pays (État de droit et reddition de comptes), de -2.5 (faible) à 2.5 (fort), selon le niveau de participation politique des femmes. La ligne horizontale correspond à 0 : les pays dont le score dépasse cette ligne sont susceptibles d'avoir pris des mesures de discrimination positive renforçant la participation des femmes à la gouvernance.

Source : OCDE (2014a), Base de données Égalité homme-femme, Institutions et Développement, http://stats.oecd.org ; Banque mondiale (s.d.), Indicateurs de gouvernance mondiaux, http://databank.worldbank.org. 
L'expérience du Sénégal montre le potentiel qu'ont les quotas de faire progresser rapidement la représentation des femmes au parlement national lorsqu'il existe également une volonté politique. Le nombre de sièges parlementaires occupés par des femmes est passé de 23 \% en 2012 à 43 \% en 2013, à la suite de la réforme de la loi électorale de 2012. Cette loi impose à toutes les listes électorales de respecter la parité femmes-hommes et de présenter hommes et femmes de manière alternée. Cependant, les quotas n’ont généralement pas d'impact majeur s'ils ne sont pas accompagnés d'autres mesures plus larges relatives au respect de la femme dans les institutions sociales (Obamamoye, 2016).

\section{Droits sur le lieu de travail}

Dans de nombreux pays, les femmes font encore face à des discriminations légales restreignant leur capacité à travailler de nuit (comme en Sierra Leone) ou à limiter ou interdire leur travail dans les mines (comme au Tchad) ou les usines (comme au Bénin) (Banque mondiale, 2015). Globalement, 60 \% des pays ouest-africains ont plus de 9 dispositions légales restreignant la capacité des femmes à obtenir un emploi au même titre qu'un homme (Graphique 14). En Mauritanie, aucune loi ne protège les femmes contre la discrimination à l'embauche ou le harcèlement sexuel sur le lieu de travail.

\section{Graphique 14}

Stéréotypes à l'encontre du travail des femmes et restrictions légales sur leur accès à l'emploi

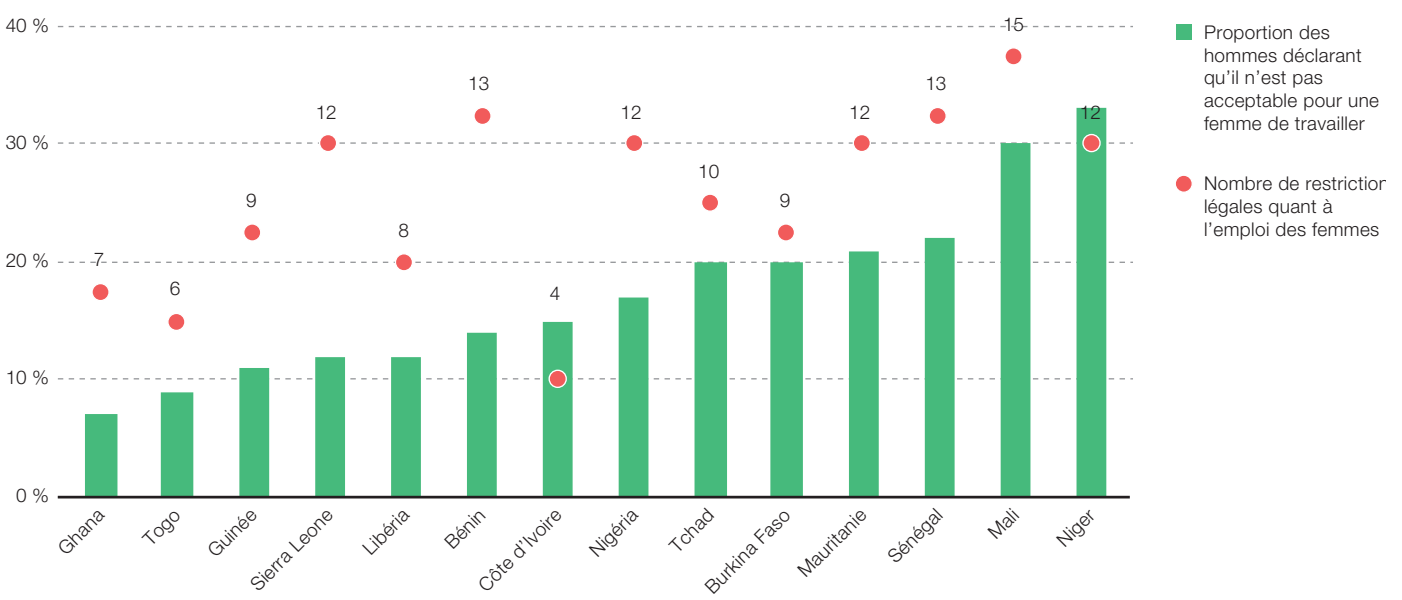

Note : Ce graphique présente la proportion d'hommes pour qui il est inacceptable que les femmes de leur famille occupent un emploi rémunéré hors de la maison et le nombre de restrictions légales quant à l'emploi des femmes, par pays.

Source : Gallup et OIT (2017), Vers un meilleur avenir pour les femmes au travail : ce qu'en pensent les femmes et les hommes, www.ilo.org/global/publications/books/WCMS_607486/lang--fr/index.htm ; Banque mondiale (2015), Women, Business and the Law 2016: Getting to Equal, http://wbl.worldbank.org/.

De plus, les pratiques et les attitudes discriminatoires concernant l'accès des femmes à l'emploi perdurent. Dans le secteur privé nigérian, de nombreux employeurs forcent les jeunes femmes à signer des contrats de travail stipulant qu'elles ne doivent pas tomber enceintes pendant les trois premières années de leur emploi. Les hommes ouest-africains sont 17 \% à penser qu'il est inacceptable qu'une femme travaille hors de la maison, et $21 \%$ préféreraient que les femmes de leur famille restent à la maison plutôt que d'occuper un emploi rémunéré (Gallup et OIT, 2017).

Avancée positive, certains gouvernements renforcent leur engagement en faveur de l'égalité sur le lieu de travail. La Côte d'Ivoire, le Mali et la Sierra Leone ont adopté des lois instituant l'égalité de rémunération pour un travail de valeur égale. En Côte d'Ivoire, le gouvernement a adopté plusieurs mesures pour accroître la participation des femmes à la population active, notamment des initiatives de renforcement des capacités 
des femmes responsables de coopératives à travers des formations aux méthodes de direction et de gestion (République de Côte d'Ivoire, 2014). Le gouvernement guinéen a mis en œuvre un programme visant à féminiser l'administration publique et mène des actions de sensibilisation pour faciliter l'accès des femmes à des postes à responsabilités (MASPFE, 2014).

\section{NOTES}

1 Cette note couvre les 15 pays membres de la Communauté économique des États de l'Afrique de l'Ouest (Bénin, Burkina Faso, Cabo Verde, Côte d'Ivoire, Gambie, Ghana, Guinée, Guinée-Bissau, Libéria, Mali, Niger, Nigéria, Sénégal, Sierra Leone et Togo), plus le Tchad et la Mauritanie.

2 Aucune section n'est consacrée au sous-indicateur "préférence pour les garçons ", car les données n'indiquent pas de préférences liées à la fertilité en Afrique de l'Ouest. Cependant, la section sur le sous-indicateur " atteintes à l'intégrité physique " comprend une analyse de la variable "femmes manquantes " afin de souligner ce phénomène dans ces pays.

3 "Parvenir à l'égalité des sexes et autonomiser toutes les femmes et les filles ».

4 Burkina Faso, Cabo Verde, Côte d'Ivoire, Gambie, Guinée, Mali et Sénégal.

5 Burkina Faso, Cabo Verde, Guinée, Mauritanie, Niger, Sénégal et Sierra Leone.

6 Le Cabo Verde n'était pas inclus dans l'édition 2014 du SIGI. II le sera dans la prochaine édition, à paraître en 2018.

7 Burkina Faso, Côte d'Ivoire, Gambie, Ghana, Libéria, Mali, Mauritanie, Niger, Nigéria, Sénégal et Tchad.

8 Ces moyennes ne tiennent pas compte du Cabo Verde, de la Gambie, de la Guinée-Bissau et du Libéria.

9 Ces deux moyennes portent sur les 17 pays. 


\section{RÉFÉRENCES}

Allen, T., P. Heinrigs et I. Heo (2018), “Agriculture, alimentation et emploi en Afrique de l'Ouest », Notes ouest-africaines, $N^{\circ} 14$, Éditions OCDE, Paris, à paraître.

Alkire, S. et al. (2013), The Women's Empowerment in Agriculture Index, Oxford Poverty and Human Development Initiative, document de travail $N^{\circ} 58$

BAD (2013), "Côte d’Ivoire : Bâtir la paix \& la cohésion sociale en luttant contre les violences faites aux femmes au sortir de la guerre ", Banque africaine de développement.

BAD/OCDE/PNUD (2017), Perspectives économiques en Afrique 2017 : Entrepreneuriat et industrialisation, Éditions de l'OCDE, Paris, http://dx.doi.org/10.1787/aeo-2017-fr.

Banque mondiale (2017), "Time and money: a study of labor constraints for female cotton producers in Côte d'Ivoire", Banque mondiale, Washington, DC.

Banque mondiale (2015), Women Business and the Law 2016: Getting to Equal, Banque mondiale, Washington, DC.

Banque mondiale (2014a), Levelling the field: Improving opportunities for women farmers in Africa, Banque mondiale, Washington, DC.

Banque mondiale (2014b), Global Financial Inclusion Database, Banque mondiale, Washington, DC, http://databank.worldbank.org/data/reports.aspx?source=1228 (consulté en octobre 2017).

Banque mondiale (2012), Rapport sur le développement dans le monde 2012 : Égalité des genres et développement, Banque mondiale, Washington, DC.

Banque mondiale (s.d.), Indicateurs du développement dans le monde (base de données), Banque mondiale, Washington, DC, http://info.worldbank.org/governance/wgi/index.aspx\#home (consulté en octobre 2017).

Banque mondiale (s.d.), World Development Indicators (database), World Bank, Washington, DC, http://data.worldbank.org/indicator (accessed October 2017).

Centre de développement de I'OCDE (2014), Social Institutions and Gender Index Synthesis Report, Éditions OCDE, Paris.

Commission de l'immigration et du statut de réfugié du Canada (2013), «Libéria : information sur la situation des femmes célibataires ou divorcées qui vivent seules, particulièrement à Monrovia ; information indiquant si elles peuvent obtenir un emploi et un logement ; services de soutien qui leur sont offerts ", Canada, http://www.refworld.org/docid/51d41ef94.html.

Comité des Nations Unies pour l'élimination de la discrimination à l'égard des femmes (CEDAW) (2014), "List of issues and questions in relation to the combined third to seventh periodic reports of Senegal », 17 novembre 2014.

CUA (2015), Agenda 2063 : L'Afrique que nous voulons, Commission de l'Union africaine, Addis Abeba.

Cuberes, D. et M. Teignier (2013), Aggregate Costs of Gender Gaps in the Labor Market: A Quantitative Estimate, Clark University, pp. 1-26.

Département d'État des États-Unis (2017), 2016 Country Reports on Human Rights Practices, United States Department of State, Bureau of Democracy, Human Rights and Labor.

Département d'État des États-Unis (2012), « 2012 Country Reports on Human Rights: Lesotho », United States Department of State, Bureau of Democracy, Human Rights and Labor.

Doss, C. et al. (2013), "Gender inequalities in ownership and control of land in Africa. Myth versus reality ", IFPRI Discussion Paper 01308.

EDS (s.d.), Enquêtes démographiques et de santé (base de données), The DHS Program, Rockville, www.statcompiler.com/en/ (consulté en octobre 2017).

FAO (2014), La situation mondiale de l'alimentation et de l'agriculture 2014, Organisation des Nations Unies pour l'alimentation et l'agriculture, Rome.

FAO (2011), La situation mondiale de l'alimentation et de l'agriculture 2010-11, Organisation des Nations Unies pour l'alimentation et l'agriculture, Rome.

Ferrant, G., A. Kolev et C. Tassot (2017), «The pursuit of happiness: Does gender parity in social institutions matter? ", OECD Development Centre Working Papers No. 337, OCDE, Paris, http://dx.doi.org/10.1787/f7e0c69c-en.

Ferrant, G. et A. Kolev, (2016), «Does gender discrimination in social institutions matter for longterm growth? Cross-country evidence ", OECD Development Centre Working Papers No. 330, OECD, Paris, http://dx.doi.org/10.1787/5jm2hz8dgls6-en.

Ferrant, G. et K. Nowacka (2015), « Measuring the drivers of gender inequality and their impact on development: the role of discriminatory social institutions ", Gender and Development, Vol. 23, $\mathrm{n}^{\circ} 2$, pp. 319-332.

Ferrant, G., L. M. Pesando et K. Nowacka (2014), Unpaid Care Work: The Missing Link in the Analysis of Gender Gaps in Labour Outcomes, Centre de développement de l'OCDE, Paris. 
Gallup et OIT (2017), Vers un meilleur avenir pour les femmes au travail : ce qu'en pensent les femmes et les hommes, Gallup, Inc. et Organisation internationale du travail.

GEM (2017), Global Entrepreneurship Monitor 2016/17 Global Report, Global Entrepreneurship Monitor.

GEM (2016), Global Entrepreneurship Monitor 2015/16 Global Report, Global Entrepreneurship Monitor.

GEM (2014), Global Entrepreneurship Monitor 2013 Global Report, Global Entrepreneurship Monitor.

Girls not brides (n.d.), Bénin, www.girlsnotbrides.org/child-marriage/benin/.

ISU (s.d.), Éducation : Participation, Base de données de l'Institut de statistiques, http://data.uis. unesco.org/ (consulté en octobre 2017).

Jones, N., C. Harper et C. Watson (2010), Stemming Girls' Chronic Poverty: Catalysing Development Change by Building Just Social Institutions, Chronic Poverty Research Centre, Manchester.

Ministère de l'action sociale, de la promotion des femmes et de l'enfance (MASPFE) (2014), Rapport de la République de Guinée sur l'évaluation de la mise en oeuvre du programme d'action de Beijing+20, Guinée.

Ministère de la femme, de l'action sociale et de la solidarité nationale [MFASSN] (2015), Feuille de route de lutte contre le mariage des enfants et les mutilations génitales féminines, Burkina Faso.

Ministère de la solidarité, de la famille, de la femme et de l'enfant (MSFFE) (2014), Document de stratégie nationale de lutte contre les violences basées sur le genre, Côte d’lvoire.

Nations Unies (2015a), "Extrême pauvreté et droits de l'homme » Note du Secrétaire général, Soixante-huitième session de l'Assemblée générale des Nations Unies, A/68/293.

Nations Unies (2015b), Trends in Contraceptive Use Worldwide 2015, Nations Unies, Département des affaires économiques et sociales, Division de la population.

Nations Unies (2015c), Transformer notre monde : le Programme de développement durable à I'horizon 2030, Assemblée générale des Nations Unies, Résolution adoptée le 25 septembre 2015.

Obamamoye, B. F. (2016), «ECOWAS and Women Representation in West Africa », Journal of Political Sciences \& Public Affairs.

OCDE (2018), Étude pays SIGI Burkina Faso, OCDE, Paris.

OCDE (2014a), Base de données Égalité homme-femme, Institutions et Développement http://stats.oecd.org (consulté en octobre 2017).

OCDE (2014b), Indicateur «Institutions sociales et hommes-femmes (SIGI), Country Profiles, http://genderindex.org.

OIT (2014), La maternité et la paternité au travail : législation et pratique dans le monde, Organisation internationale du travail, Genève.

OIT (s.d.), Ratifications de C183 - Convention (No. 183) sur la protection de la maternité, www.ilo.org/dyn/normlex/en/f?p=NORMLEXPUB:11300:0::NO::P11300_INSTRUMENT_ ID:312328 (consulté en octobre 2017).

OMS (2017), « La violence à l'encontre des femmes », Organisation mondiale de la santé, www.who.int/.

ONU Femmes (2016), Le progrès des femmes dans le monde 2015-2016, ONU Femmes, États-Unis.

ONU Femmes (2015), Preventing Conflict, Transforming Justice, Securing the Peace, ONU Femmes, États-Unis.

O'Sullivan, M. et al. (2014), Levelling the field: improving opportunities for women farmers in Africa, Groupe de la Banque mondiale, Washington, DC.

Sonke Gender Justice Network et MenEngage (2009), Working with men and boys: Emerging strategies from across Africa to address Gender-based Violence and HIVIAIDS, Sonke Gender Justice Network and MenEngage Network, Johannesbourg.

UIP (2017), Women in politics, nion interparlementaire et ONU Femmes, Genève.

UNFPA (2012), Programme conjoint UNFPA-UNICEF sur les mutilations génitales féminines/ l'excision: Accélérer le changement, UNFPA-UNICEF, New York.

UNICEF (2015), Mariages d'enfants, grossesses précoces et formation de la famille en Afrique de l'Ouest et du Centre, Fonds des Nations Unies pour l'enfance, New York.

UNICEF (2013), Mutilations génitales féminines/excision: Bilan statistique et examen des dynamiques du changement, Fonds des Nations Unies pour l'enfance, New York.

van den Bold, M. et al. (2015), "Can integrated Agriculture-Nutrition Programmes Change Gender Norms on Land and Asset Ownership? Evidence from Burkina Faso ", The Journal of Development Studies, vol. 51, n 9, pp. 1155-1174. 


\section{ANNEXE 1 : À PROPOS DU SIGI}

L'indicateur SIGI (Institutions sociales et égalité femmes-hommes) mesure les discriminations qui s'exercent contre les femmes au sein des institutions sociales de 160 pays. Le SIGI comprend des fiches pays, un classement des pays et la base de données Égalité hommefemme, Institutions et Développement. Il constitue un outil de recherche et de plaidoyer politique pour la communauté du développement et pour les décideurs politiques.

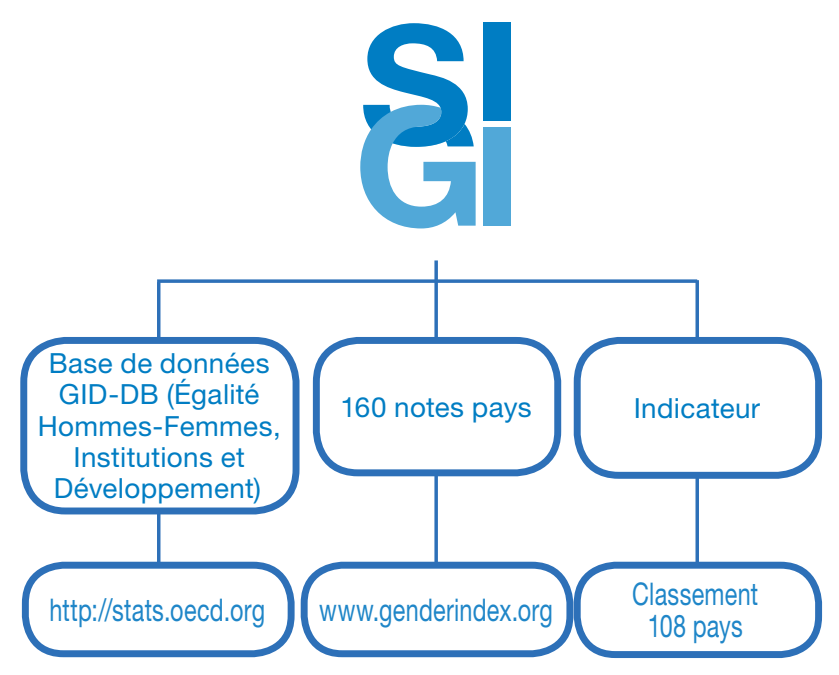

Note : En tant qu'indicateur composite, le SIGl évalue le niveau de discrimination des pays à travers 14 indicateurs, agrégés en cinq dimensions. Le SIGI varie de 0 (absence d'inégalité) à 1 (inégalité totale). Le SIGl classe les pays en cinq groupes en fonction de leur niveau de discrimination, s'échelonnant de très faibles à très élevés.

Le SIGI mesure les institutions sociales discriminatoires à travers cinq dimensions englobant les principaux domaines socio-économiques qui affectent les femmes au cours de leur cycle de vie : les discriminations au sein du code de la famille, les atteintes à l'intégrité physique, la préférence pour les garçons, l'accès restreint aux ressources et aux biens, et les atteintes aux libertés civiles. 


\section{ANNEXE 2 : RÉSULTATS PAR SOUS-INDICATEUR DU SIGI POUR LES PAYS D'AFRIQUE DE L'OUEST}

\begin{tabular}{|c|c|c|c|c|c|c|c|c|c|c|c|}
\hline Pays & SIGI & \multicolumn{2}{|c|}{$\begin{array}{l}\text { Discriminations au } \\
\text { sein du code de la } \\
\text { famille }\end{array}$} & \multicolumn{2}{|c|}{$\begin{array}{c}\text { Atteintes à } \\
\text { l'intégrité physique }\end{array}$} & \multicolumn{2}{|c|}{$\begin{array}{l}\text { Préférence pour } \\
\text { les garçons }\end{array}$} & \multicolumn{2}{|c|}{$\begin{array}{l}\text { Accès restreint } \\
\text { aux ressources et } \\
\text { aux biens }\end{array}$} & \multicolumn{2}{|c|}{$\begin{array}{c}\text { Atteintes aux libertés } \\
\text { civiles }\end{array}$} \\
\hline \multicolumn{12}{|c|}{ Niveaux intermédiaires de discrimination dans Ie SIGI } \\
\hline Togo & 0.1860 & 0.3696 & $\begin{array}{c}\text { inter- } \\
\text { médiaires }\end{array}$ & 0.5488 & élevés & 0.1326 & $\begin{array}{c}\text { inter- } \\
\text { médiaires }\end{array}$ & 0.5913 & élevés & 0.3539 & $\begin{array}{l}\text { inter- } \\
\text { médiaires }\end{array}$ \\
\hline Sénégal & 0.1985 & 0.5931 & $\begin{array}{l}\text { très } \\
\text { élevés }\end{array}$ & 0.6337 & élevés & 0.0888 & faibles & 0.4076 & $\begin{array}{c}\text { inter- } \\
\text { médiaires }\end{array}$ & 0.2554 & faibles \\
\hline Guinée-Bissau & 0.2110 & 0.4073 & $\begin{array}{c}\text { inter- } \\
\text { médiaires }\end{array}$ & 0.4932 & élevés & 0.0713 & faibles & 0.5913 & élevés & 0.5399 & élevés \\
\hline \multicolumn{12}{|c|}{ Niveaux élevés de discrimination dans le SIGI } \\
\hline Côte d'Ivoire & 0.2537 & 0.4955 & élevés & 0.5895 & élevés & 0.1858 & $\begin{array}{l}\text { inter- } \\
\text { médiaire }\end{array}$ & 0.5913 & élevés & 0.5399 & élevés \\
\hline Bénin & 0.2780 & 0.2763 & $\begin{array}{c}\text { inter- } \\
\text { médiaires }\end{array}$ & 0.4432 & élevés & 0.3677 & élevés & 0.5913 & élevés & 0.7953 & très élevés \\
\hline Burkina Faso & 0.2819 & 0.5419 & élevés & 0.7257 & $\begin{array}{l}\text { très } \\
\text { élevés }\end{array}$ & 0.1910 & inter- & 0.5913 & élevés & 0.4505 & $\begin{array}{l}\text { inter- } \\
\text { médiaires }\end{array}$ \\
\hline Ghana & 0.2988 & 0.3946 & $\begin{array}{c}\text { inter- } \\
\text { médiaires }\end{array}$ & 0.5491 & élevés & 0.3136 & élevés & 0.8044 & $\begin{array}{l}\text { très } \\
\text { élevés }\end{array}$ & 0.5399 & élevés \\
\hline Guinée & 0.3206 & 0.5413 & élevés & 0.9515 & $\begin{array}{l}\text { très } \\
\text { élevés }\end{array}$ & 0.2253 & $\begin{array}{l}\text { inter- } \\
\text { médiaires }\end{array}$ & 0.3885 & $\begin{array}{l}\text { intermé- } \\
\text { diaire }\end{array}$ & 0.4505 & $\begin{array}{l}\text { inter- } \\
\text { médiaires }\end{array}$ \\
\hline \multicolumn{12}{|c|}{ Niveaux très élevés de discrimination dans le SIGI } \\
\hline Sierra Leone & 0.3720 & 0.3309 & $\begin{array}{l}\text { inter- } \\
\text { médiaires }\end{array}$ & 0.8550 & $\begin{array}{l}\text { très } \\
\text { élevés }\end{array}$ & 0.0364 & $\begin{array}{l}\text { très } \\
\text { faibles }\end{array}$ & 0.8044 & $\begin{array}{l}\text { très } \\
\text { élevés }\end{array}$ & 0.6093 & élevés \\
\hline Libéria & 0.3828 & 0.5669 & $\begin{array}{l}\text { très } \\
\text { élevés }\end{array}$ & 0.8907 & $\begin{array}{l}\text { très } \\
\text { élevés }\end{array}$ & 0.0236 & $\begin{array}{l}\text { très } \\
\text { faibles }\end{array}$ & 0.4076 & $\begin{array}{c}\text { inter- } \\
\text { médiaire }\end{array}$ & 0.7953 & très élevés \\
\hline Nigéria & 0.3911 & 0.6723 & $\begin{array}{l}\text { très } \\
\text { élevés }\end{array}$ & 0.4766 & élevés & 0.2494 & élevés & 0.7626 & $\begin{array}{l}\text { très } \\
\text { élevés }\end{array}$ & 0.7953 & très élevés \\
\hline Mauritanie & 0.3954 & 0.7556 & $\begin{array}{l}\text { très } \\
\text { élevés }\end{array}$ & 0.9939 & $\begin{array}{l}\text { très } \\
\text { élevés }\end{array}$ & 0.1746 & $\begin{array}{l}\text { inter- } \\
\text { médiaires }\end{array}$ & 0.5913 & élevés & 0.1951 & faibles \\
\hline Niger & 0.4415 & 1.0000 & $\begin{array}{l}\text { très } \\
\text { élevés }\end{array}$ & 0.4059 & $\begin{array}{l}\text { inter- } \\
\text { médiaire }\end{array}$ & 0.1746 & $\begin{array}{c}\text { inter- } \\
\text { médiaires }\end{array}$ & 0.5913 & élevés & 0.8140 & très élevés \\
\hline Tchad & 0.4665 & 0.9705 & $\begin{array}{l}\text { très } \\
\text { élevés }\end{array}$ & 0.8185 & $\begin{array}{l}\text { très } \\
\text { élevés }\end{array}$ & 0.0014 & $\begin{array}{l}\text { très } \\
\text { faibles }\end{array}$ & 0.5913 & élevés & 0.6093 & élevés \\
\hline Mali & 0.5164 & 0.8309 & $\begin{array}{l}\text { très } \\
\text { élevés }\end{array}$ & 1.0000 & $\begin{array}{l}\text { très } \\
\text { élevés }\end{array}$ & 0.3048 & élevés & 0.4076 & $\begin{array}{c:}\text { inter- } \\
\text { médiaire }\end{array}$ & 0.7953 & très élevés \\
\hline Gambie & 0.5240 & 0.5131 & élevés & 0.8509 & $\begin{array}{l}\text { très } \\
\text { élevés }\end{array}$ & 0.0000 & $\begin{array}{l}\text { très } \\
\text { faibles }\end{array}$ & 1.0000 & $\begin{array}{l}\text { très } \\
\text { élevés }\end{array}$ & 0.7953 & très élevés \\
\hline \multicolumn{12}{|c|}{ Non classé dans le SIGI } \\
\hline Cabo Verde & n. a. & n. a. & & n. a. & & n. a. & & n. a. & & n. a. & \\
\hline
\end{tabular}

*n.a.: not available/non disponible 


\section{ANNEXE 3 : RATIFICATIONS DES PROTOCOLES MONDIAUX ET RÉGIONAUX SUR L'ÉGALITÉ FEMMES-HOMMES}

\begin{tabular}{|c|c|c|c|c|c|c|}
\hline Pays & $\begin{array}{l}\text { Convention sur } \\
\text { l'élimination de } \\
\text { toutes les formes } \\
\text { de discrimination } \\
\text { à l'égard des } \\
\text { femmes (CEDEF) }\end{array}$ & $\begin{array}{l}\text { Protocole facultatif } \\
\text { à la Convention sur } \\
\text { l'élimination de } \\
\text { toutes les formes } \\
\text { de discrimination à } \\
\text { l'égard des femmes }\end{array}$ & $\begin{array}{l}\text { Protocole à la Charte } \\
\text { africaine des droits de } \\
\text { I'homme et des } \\
\text { peuples relatif aux } \\
\text { droits des femmes } \\
\text { (Protocole de Maputo) }\end{array}$ & $\begin{array}{l}\text { Charte africaine } \\
\text { des droits et du } \\
\text { bien-être de } \\
\text { l'enfant }\end{array}$ & $\begin{array}{l}\text { Charte africaine } \\
\text { de la jeunesse }\end{array}$ & $\begin{array}{c}\text { C183 - Convention } \\
\left(n^{\circ} 183\right) \text { sur la } \\
\text { protection de la } \\
\text { maternité }\end{array}$ \\
\hline Bénin & Ratifiée & Signé & Ratifié & Ratifiée & Signée & Ratifiée \\
\hline Burkina Faso & Ratifiée & Ratifié & Ratifié & Ratifiée & Ratifiée & Ratifiée \\
\hline Cabo Verde & Ratifiée & Ratifié & Ratifié & Ratifiée & n.a. & n.a. \\
\hline Côte d'Ivoire & Ratifiée & Ratifié & Ratifié & Ratifiée & Ratifiée & n.a. \\
\hline Gambie & Ratifiée & n.a. & Ratifié & Ratifiée & Ratifiée & n.a. \\
\hline Ghana & Ratifiée & Ratifié & Ratifié & Ratifiée & Signée & n.a. \\
\hline Guinée & Ratifiée & n.a. & Ratifié & Ratifiée & Signée & n.a. \\
\hline Guinée-Bissau & Ratifiée & Ratifié & Ratifié & Signée & Ratifiée & n.a. \\
\hline Libéria & Ratifiée & Signé & Ratifié & Signée & Signée & n.a. \\
\hline Mali & Ratifiée & Ratifié & Ratifié & Ratifiée & Ratifiée & Ratifiée \\
\hline Mauritanie & Ratifiée & n.a. & Ratifié & Ratifiée & n.a. & n.a. \\
\hline Niger & Ratifiée & Ratifié & Signé & Ratifiée & Ratifiée & n.a. \\
\hline Nigéria & Ratifiée & Ratifié & Ratifié & Ratifiée & Ratifiée & n.a. \\
\hline Sénégal & Ratifiée & Ratifié & Ratifié & Ratifiée & Ratifiée & $\begin{array}{l}\text { En vigueur le } \\
18 \text { avril } 2018\end{array}$ \\
\hline Sierra Leone & Ratifiée & Signé & Signé & Ratifiée & Signée & n.a. \\
\hline Tchad & Ratifiée & Signé & Ratifié & Ratifiée & Signée & n.a. \\
\hline Togo & Ratifiée & n.a. & Ratifié & Ratifiée & Ratifiée & n.a. \\
\hline
\end{tabular}




\section{GLOSSAIRE}

Les institutions sociales fixent les paramètres au regard desquels les décisions, les choix ou les comportements sont jugés acceptables ou inacceptables pour les femmes et les hommes dans une société donnée. Elles définissent ainsi les rôles des femmes et des hommes et influent sur leurs opportunités.

Les institutions sociales discriminatoires sont les lois formelles et informelles, les normes et les pratiques sociales qui entravent les droits des femmes ou les excluent, et par conséquent limitent leur accès à la justice, aux ressources et aux opportunités.

Le mariage précoce est un mariage formel ou une union informelle contracté(e) avant l'âge minimum recommandé de 18 ans défini dans les déclarations et conventions internationales.

Le travail de soin non rémunéré désigne les activités domestiques non rémunérées, telles que les soins aux enfants, la collecte d'eau et de bois et les travaux ménagers. 




\section{DANS LA MÊME COLLECTION :}

Allen, T. et P. Heinrigs (2016), (c Les nouvelles opportunités de l'économie alimentaire ouest-africaine », Notes ouest-africaines, N¹, Éditions OCDE, Paris. http://dx.doi.org/10.1787/5jlwjg67l25f-fr

Lewis, K. and C. Buontempo (2016), « Climate Impacts in the Sahel and West Africa: The Role of Climate Science in Policy Making ), Notes ouest-africaines, N², Éditions OCDE, Paris. http://dx.doi.org/10.1787/5jlsmktwjcd0-en

Gnisci, D. (2016), “ Women's Roles in the West African Food System: Implications and Prospects for Food Security and Resilience m, Notes ouest-africaines, N³, Éditions OCDE, Paris. http://dx.doi.org/10.1787/5jlpl4mh1hxn-en

Staatz, J. and F. Hollinger (2016), (c West African Food Systems and Changing Consumer Demands ), Notes ouest-africaines, N4, Éditions OCDE, Paris. http://dx.doi.org/10.1787/b165522b-en

Prieto Curiel, R., P. Heinrigs and I. Heo (2017), « Cities and Spatial Interactions in West Africa: A Clustering Analysis of the Local Interactions of Urban Agglomerations m, Notes ouest-africaines, N5, Éditions OCDE, Paris. http://dx.doi.org/10.1787/57b30601-en

Walther, O. (2017), ( Les réseaux de la coopération transfrontalière en Afrique de l'Ouest ), Notes ouest-africaines, N06, Éditions OCDE, Paris. http://dx.doi.org/10.1787/b7ad4957-fr

Ibrahim, I.Y. (2017), “ Insurrections jihadistes en Afrique de l’Ouest : idéologie mondiale, contexte local, motivations individuelles m, Notes ouest-africaines, N07, Éditions OCDE, Paris. http://dx.doi.org/10.1787/1da64565-fr

Allen, T. (2017), ( Le coût des prix alimentaires élevés en Afrique de l'Ouest ), Notes ouest-africaines, N08, Éditions OCDE, Paris. http://dx.doi.org/10.1787/48e99091-fr

van Den Hoek, J. (2017), « Agricultural market activity and Boko Haram attacks in northeastern Nigeria ), Notes ouest-africaines, Nº9, Éditions OCDE, Paris. http://dx.doi.org/10.1787/13ba9f2e-en

Walther, O. (2017), (c Guerres et conflits au Sahara-Sahel ), Notes ouest-africaines, N¹0, Éditions OCDE, Paris. https://doi.org/10.1787/8bbc5813-en

Elisher, S. (2018), « Defying the Odds? Nigerien Responses to Foreign and Domestic Security Challenges »), Notes ouest-africaines, N¹1, Éditions OCDE, Paris. https://doi.org/10.1787/104d1c6d-en

Eizenga, D. (2018), (c The Unstable Foundations of Political Stability in Chad ), Notes ouest-africaines, N¹2, Éditions OCDE, Paris. https://doi.org/10.1787/508844d3-en

En savoir plus : lia.beyeler@oecd.org 